\title{
Self-dual tensors and partial supersymmetry breaking in five dimensions
}

\author{
Thomas W. Grimm and Andreas Kapfer \\ Max-Planck-Institut für Physik, \\ Föhringer Ring 6, 80805 Munich, Germany \\ E-mail: grimm@mpp.mpg.de, kapfer@mpp.mpg.de
}

ABSTRACT: We study spontaneous supersymmetry breaking of five-dimensional supergravity theories from sixteen to eight supercharges in Minkowski vacua. This $\mathcal{N}=4 \rightarrow \mathcal{N}=2$ breaking is induced by Abelian gaugings that require the introduction of self-dual tensor fields accompanying some of the vectors in the gravity and vector multiplets. These tensor fields have first-order kinetic terms and become massive by a Stückelberg-like mechanism. We identify the general class of $\mathcal{N}=2$ vacua and show how the $\mathcal{N}=4$ spectrum splits into massless and massive $\mathcal{N}=2$ multiplets. In particular, we find a massive gravitino multiplet, with two complex massive tensors, and a number of massive tensor multiplets and hypermultiplets. We determine the resulting $\mathcal{N}=2$ effective action for the massless multiplets obtained by integrating out massive fields. We show that the metric and Chern-Simons terms of the vectors are corrected at one-loop by massive tensors as well as spin- $1 / 2$ and spin-3/2 fermions. These contributions are independent of the supersymmetry-breaking scale and thus have to be included at arbitrarily low energies.

KEYwords: Supersymmetry Breaking, Effective field theories, Supersymmetric Effective Theories

ArXiv ePrint: 1402.3529 


\section{Contents}

1 Introduction 1

2 Gauged $\mathcal{N}=4$ supergravity in five dimensions 3

2.1 Generalities 3

$\begin{array}{lll}2.2 & \text { Vacuum conditions and supersymmetry breaking } & 7\end{array}$

3 Supergravity breaking from $\mathcal{N}=4$ to $\mathcal{N}=2 \quad 9$

3.1 Solution to the $\mathcal{N}=2$ condition $\quad 9$

$\begin{array}{lll}3.2 & \text { The tensorial Higgs mechanism } & 10\end{array}$

$\begin{array}{lll}3.3 & \text { The super-Higgs mechanism } & 13\end{array}$

4 Mass spectrum and effective action $\quad \mathbf{1 5}$

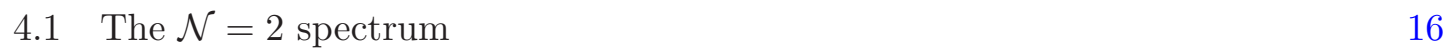

$\begin{array}{ll}4.2 \text { General } \mathcal{N}=2 \text { action and classical matching } & 18\end{array}$

$\begin{array}{lll}4.3 & \text { One-loop effects and Chern-Simons terms } & 21\end{array}$

5 Conclusions $\quad 22$

A Conventions and identities $\quad 23$

$\begin{array}{lll}\text { A.1 Spacetime conventions } & 23\end{array}$

$\begin{array}{lll}\text { A.2 } & \mathrm{SO}(5) \text { gamma matrices } & 25\end{array}$

B Derivation of the mass terms and couplings $\quad 25$

B.1 Fermion masses 26

$\begin{array}{ll}\text { B.2 Scalar masses } & 27\end{array}$

\section{Introduction}

A systematic classification of supersymmetric vacua of supergravity theories in various dimensions has been a challenge since the first constructions of such theories. Supergravity theories with non-minimal supersymmetry can often admit Minkowski or anti-de Sitter ground states that preserve only a partial amount of supersymmetry. Finding such solutions is typically more involved than determining the fully supersymmetric solutions. For supergravity theories formulated in even space-time dimensions various breaking patterns have been investigated in detail. For example, the $\mathcal{N}=2$ to $\mathcal{N}=1$ breaking in fourdimensional supergravity theories has been investigated already in [1-8]. Recently, there has been a renewed interest in this direction [9-11], which was partially triggered by the application to flux compactifications of string theory [12]. The general analysis of [10] heavily employs the powerful techniques provided by the embedding tensor formalism [13, 14]. 
The general study of partial supersymmetry breaking in odd-dimensional theories has attracted much less attention. Such theories, however, possess the interesting new possibility that the dynamics of some fields can arise from Chern-Simons-type couplings that are topological in nature. As was pointed out already for three-dimensional supergravity theories [15] such couplings can allow for special supersymmetry breaking patterns. In this work we show that in five-dimensional supergravity theories with sixteen supercharges, denoted as $\mathcal{N}=4$, Chern-Simons-type couplings for two-from tensor fields can yield interesting new supersymmetry breaking patterns to vacua preserving eight supercharges, denoted as $\mathcal{N}=2$. Such tensor fields can have first-order kinetic terms and become massive by a Stückelberg-like mechanism in which they eat a dynamical vector field [16-18]. The degrees of freedom of such tensors are counted by realizing that they have zero degrees of freedom before eating the vector, but admit three degrees of freedom as massive fields. Hence they should be distinguished from tensors with standard kinetic and mass terms. They have been named self-dual tensors in [16]. In fact, such five-dimensional self-dual tensors arise, for example, as massive Kaluza-Klein modes of a six-dimensional self-dual tensor compactified on a circle $[16,19]$. The mechanism rendering the tensor fields massive by eating a vector will be called tensorial Higgs mechanism in the following.

In general, the couplings of the five-dimensional self-dual tensors to the vector fields and the form of the first order kinetic terms are encoded by a constant anti-symmetric matrix $\xi_{M N}$, known as the embedding tensor [17, 18]. A non-trivial $\xi_{M N}$ also induces vector gaugings and a scalar potential. We analyze the conditions on $\xi_{M N}$ that yield partial supersymmetry breaking to an $\mathcal{N}=2$ Minkowski vacuum. The massless and massive $\mathcal{N}=2$ spectrum comprising fluctuations around this vacuum are then determined systematically. We particularly stress the appearance of massive tensor fields and massive spin- $1 / 2$ and spin- $3 / 2$ fermions. This allows us to derive the key features of the effective $\mathcal{N}=2$ supergravity theory arising for the massless fluctuations around the ground state.

The $\mathcal{N}=2$ effective action for the massless fields comprises of two parts. Firstly, there are the classical couplings inherited from the underlying $\mathcal{N}=4$ theory. They are determined by truncating the original theory to the appropriately combined massless modes. At energy scales far below the supersymmetry breaking scale one might have expected that this determined already the complete $\mathcal{N}=2$ theory. However, as we show in detail in this work, the massive tensor, spin- $1 / 2$ and spin-3/2 modes have to actually be integrated out and induce non-trivial corrections. In fact, using the results of [20], one infers that if these massive fields are charged under some vector field, they induce non-trivial one-loop corrections to the Chern-Simons terms for the vector. One-loop corrections to the Chern-Simons terms due to massive charged spin- $1 / 2$ fermions have been considered in [21, 22], but we stress here that in the $\mathcal{N}=4$ to $\mathcal{N}=2$ breaking both massive tensors and gravitini alter the result crucially. All these one-loop corrections are independent of the mass scale of these fields and therefore have to be taken into account in a consistent effective theory at scales well below the supersymmetry breaking scale.

Five-dimensional partial supersymmetry breaking can also occur in string compactifications. For example, compactifications of Type IIB string theory on a certain class of five-dimensional compact spaces can yield five-dimensional $\mathcal{N}=4$ theories that admit 
half-supersymmetric vacua as discussed, for example, in [23-30]. The generic analysis of these effective theories yields the existence also of AdS vacua that are of particular interest for applications of the AdS/CFT duality. Our study of Minkowski vacua should be viewed as a crucial step in obtaining a full classification including also AdS vacua. We will see that already in the Minkowski vacua, arising due to the existence of tensor fields, we can highlight interesting features of partial supersymmetry breaking in five dimensions. In particular, we show that the $\mathcal{N}=2$ effective supergravity theory for the massless fluctuations around the $\mathcal{N}=2$ vacuum depends on the massive states of the underlying $\mathcal{N}=4$ theory in an intriguing way.

The paper is organized as follows. In section 2 we recall some general facts about $\mathcal{N}=4$ supergravity theories. For an Abelian configuration with a non-trivial embedding tensor $\xi_{M N}$ we determine the conditions for the scalar potential to admit supersymmetric vacua. Supersymmetry breaking from $\mathcal{N}=4$ to $\mathcal{N}=2$ is studied in detail in section 3 . We give a detailed account of the tensorial Higgs mechanism and the super-Higgs mechanism. In section 4 we then determine the $\mathcal{N}=2$ spectrum and one-loop effective action. We argue that all massive multiplets induce one-loop corrections to the vector couplings of the theory that are independent of the supersymmetry breaking scale. In two supplementary appendices we summarize our conventions and give details on the computation of fermion and scalar masses.

\section{Gauged $\mathcal{N}=4$ supergravity in five dimensions}

In this section we summarize some important facts about $\mathcal{N}=4$ supergravity theories. We first discuss the spectrum and action in subsection 2.1. This will also allow us to state the conventions used throughout the paper. Further conventions and useful identities are supplemented in appendix A. In subsection 2.2 we study the vacua of an $\mathcal{N}=4$ theory characterized by the components $\xi_{M N}$ of the embedding tensor and comment on the possible amounts of supersymmetries preserved in the vacuum.

\subsection{Generalities}

Let us start reviewing the general properties of $\mathcal{N}=4$ gauged supergravity in five dimensions following $[17,18] .{ }^{1}$ We first focus on ungauged Maxwell-Einstein supergravity that describes the coupling of $n$ vector multiplets and a gravity multiplet. Note that in the ungauged theory one can replace the vector multiplets by dual tensor multiplets. The gravity multiplet consists of the fields

$$
\left(g_{\mu \nu}, \psi_{\mu}^{i}, A_{\mu}^{i j}, A_{\mu}^{0}, \chi^{i}, \sigma\right)
$$

with the metric $g_{\mu \nu}$, four spin-3/2 gravitini $\psi_{\mu}^{i}$, six vectors $\left(A_{\mu}^{i j}, A_{\mu}^{0}\right)$, four spin- $1 / 2$ fermions $\chi^{i}$ and one real scalar $\sigma$. The indices $i, j=1, \ldots, 4$ run over the fundamental representation

\footnotetext{
${ }^{1}$ Let us stress again that in our conventions five-dimensional $\mathcal{N}=4$ supergravity theories have 16 supercharges and thus are half-maximal supergravities.
} 
of the R-symmetry group $\mathrm{USp}(4)$. We denote the symplectic form of $\mathrm{USp}(4)$ by $\Omega$. It has the following properties

$$
\Omega_{i j}=-\Omega_{j i}, \quad \Omega_{i j}=\Omega^{i j}, \quad \Omega_{i j} \Omega^{j k}=-\delta_{i}^{k} .
$$

Indices of USp(4) are raised and lowered according to

$$
V^{i}=\Omega^{i j} V_{j}, \quad V_{i}=V^{j} \Omega_{j i} .
$$

The 5 representation of $\mathrm{USp}(4)$ is denoted by the double index $i j$ with the following properties

$$
A_{\mu}^{i j}=-A_{\mu}^{j i}, \quad A_{\mu}^{i j} \Omega_{i j}=0, \quad\left(A_{\mu}^{i j}\right)^{*}=A_{\mu i j} .
$$

Note that $\mathrm{USp}(4)$ is the spin group of $\mathrm{SO}(5)$. We will therefore often use the local isomorphism $\mathrm{SO}(5) \cong \mathrm{USp}(4)$ to rewrite representations. We denote the indices of the fundamental representation of $\mathrm{SO}(5)$ by $m, n=1, \ldots, 5$. They are raised and lowered with the Kronecker delta $\delta_{m n}$. We stress that all massless fermions in this work are symplectic Majorana spinors. Further conventions and useful relations can be found in appendix A. Moreover we will conveniently use the definition

$$
\Sigma:=e^{\sigma / \sqrt{3}},
$$

where $\sigma$ is the real scalar in the gravity multiplet (2.1).

In addition to the gravity multiplet we have $n$ vector multiplets, labeled by the indices $a, b=1, \ldots, n$, which are raised and lowered by the Kronecker delta $\delta_{a b}$. The field content is

$$
\left(A_{\mu}^{a}, \lambda^{i a}, \phi^{i j a}\right) .
$$

The $A_{\mu}^{a}$ denote vectors, $\lambda^{i a}$ spin- $1 / 2$ fermions and the $\phi^{i j a}$ are scalars in the $\mathbf{5}$ of USp(4).

Collecting all scalars of the theory they span the coset manifold

$$
\mathcal{M}=\mathcal{M}_{5, n} \times \mathrm{SO}(1,1), \quad \mathcal{M}_{5, n}=\frac{\mathrm{SO}(5, n)}{\mathrm{SO}(5) \times \mathrm{SO}(n)},
$$

where the coset $\mathcal{M}_{5, n}$ is parametrized by the scalars $\phi^{i j a}$ in the vector multiplets, while the $\mathrm{SO}(1,1)$ factor is described by the scalar $\sigma$ in the gravity multiplet. Thus the global symmetry group of the theory is $\mathrm{SO}(5, n) \times \mathrm{SO}(1,1)$. We also introduce $\mathrm{SO}(5, n)$ indices $M, N=1, \ldots 5+n$, which are raised and lowered with the $\mathrm{SO}(5, n)$ metric $\left(\eta_{M N}\right)=$ $\operatorname{diag}(-1,-1,-1,-1,-1,+1, \ldots,+1)$. The generators $t_{M N}$ of $\mathrm{SO}(5, n)$ and $t_{0}$ of $\mathrm{SO}(1,1)$ are given $b^{2}$

$$
t_{M N P}{ }^{Q}=2 \delta_{[M}^{Q} \eta_{N] P}, \quad t_{0 M}{ }^{N}=-\frac{1}{2} \delta_{M}^{N}, \quad t_{M N 0}{ }^{0}=0, \quad t_{00}{ }^{0}=1 .
$$

To couple the vector multiplets to the gravity multiplet we note that the vectors of both kind of multiplets transform as a singlet $A^{0}$ and the fundamental representation $A^{M}$ of $\mathrm{SO}(5, n)$ :

$$
\left(A^{0}, A^{i j}, A^{n}\right) \rightarrow\left(A^{0}, A^{M}\right)
$$

with $\mathrm{SO}(1,1)$ charges -1 and $1 / 2$ for $A^{0}$ and $A^{M}$, respectively.

\footnotetext{
${ }^{2}$ All antisymmetrizations in this paper include a factor of $1 / n$ ! .
} 
The coset space $\mathcal{M}_{5, n}$ is most conveniently described by the coset representatives $\mathcal{V}=\left(\mathcal{V}_{M}{ }^{m}, \mathcal{V}_{M}{ }^{a}\right)$, where $m=1, \ldots, 5$ is the $\mathrm{SO}(5)$ index, while $a=1, \ldots n$ is the $\mathrm{SO}(n)$ index. Global $\mathrm{SO}(5, n)$ transformations on $\mathcal{V}$ act from the left, while local $\mathrm{SO}(5) \times \mathrm{SO}(n)$ transformations act from the right. We stress that

$$
\mathcal{V}_{M}{ }^{a}=\eta_{M N} \mathcal{V}^{N a}, \quad \mathcal{V}_{M}^{m}=-\eta_{M N} \mathcal{V}^{N m}
$$

Note that since $\left(\mathcal{V}_{M}{ }^{m}, \mathcal{V}_{M}{ }^{a}\right) \in \mathrm{SO}(5, n)$ we have

$$
\eta_{M N}=-\mathcal{V}_{M}{ }^{m} \mathcal{V}_{N m}+\mathcal{V}_{M}{ }^{a} \mathcal{V}_{N a}
$$

Let us also introduce a non-constant positive definite metric on the coset

$$
M_{M N}=\mathcal{V}_{M}{ }^{m} \mathcal{V}_{N m}+\mathcal{V}_{M}^{a} \mathcal{V}_{N a}
$$

The inverse of $M_{M N}$ is given by $M^{M N}$. We can also make use of the local isomorphism $\mathrm{SO}(5) \cong \mathrm{USp}(4)$ to define

$$
\mathcal{V}_{M}{ }^{i j}:=\frac{1}{2} \mathcal{V}_{M}{ }^{m} \Gamma_{m}{ }^{i j}, \quad \mathcal{V}_{M}{ }^{m}=\frac{1}{2} \mathcal{V}_{M}{ }^{i j} \Gamma^{m}{ }_{i j},
$$

where $\mathcal{V}_{M}{ }^{i j}$ transforms in the 5 of $\operatorname{USp}(4)$ and $\Gamma^{m}{ }_{i j}$ are the components of the gammamatrix $\Gamma^{m}$ of $\mathrm{SO}(5)$.

There are now different possibilities to gauge some of the global symmetries. The various gaugings can be described in terms of the embedding tensors $f_{M N P}, \xi_{M N}$ and $\xi_{M}$ that are totally antisymmetric in their indices. They specify a covariant derivative ${ }^{3}$

$$
D_{\mu}=\nabla_{\mu}-A_{\mu}^{M} f_{M}^{N P} t_{N P}-A_{\mu}^{0} \xi^{N P} t_{N P}-A_{\mu}^{M} \xi^{N} t_{M N}-A_{\mu}^{M} \xi_{M} t_{0} .
$$

We note that in the ungauged theory the embedding tensors are spurionic objects transforming under the global symmetry group. As soon as we fix a value for the tensor components, the global symmetry group is broken down to a subgroup. In this paper we will focus solely on the gauging $\xi_{M N}$, since the calculations simplify considerably. Furthermore, we will find that a non-vanishing $\xi_{M N}$ is essential in the tensorial Higgs mechanism and interesting parts of the structure arising with general gaugings are already present in the case of $f_{M N P}=\xi_{M}=0$. Therefore, we will from now on set

$$
f_{M N P}=\xi_{M}=0
$$

such that the covariant derivative (2.14) simplifies to

$$
D_{\mu}=\nabla_{\mu}-A_{\mu}^{0} \xi^{N P} t_{N P}
$$

We note that in the case of $f_{M N P}=\xi_{M}=0$ there are no further constraints on $\xi_{M N}$ except of antisymmetry. It is important to remark that such a nontrivial gauging has the effect,

\footnotetext{
${ }^{3}$ Note that one can explicitly include a gauge coupling constant $g$ whenever an embedding tensor appears. For convenience we take $g=1$ in the following.
} 
that we are forced to dualize some of the vector fields $A_{\mu}^{M}$ into two-forms $B_{\mu \nu} M$. Therefore, in order to write down the most general gauged supergravity with $f_{M N P}=\xi_{M}=0$, we have to consider an action where both $A_{\mu}^{M}$ and $B_{\mu \nu M}$ are present. In this formulation the tensor fields $B_{\mu \nu M}$ carry no on-shell degrees of freedom, they can, however, become massive by eating a dynamical vector and acquire three degrees of freedom. We will discuss this further in subsection 3.2.

The bosonic Lagrangian of this $\mathcal{N}=4$ supergravity theory reads [17, 18]

$$
\begin{aligned}
e^{-1} \mathcal{L}_{\mathrm{bos}}= & -\frac{1}{2} R-\frac{1}{4} \Sigma^{2} M_{M N} \mathcal{H}_{\mu \nu}^{M} \mathcal{H}^{\mu \nu N}-\frac{1}{4} \Sigma^{-4} F_{\mu \nu}^{0} F^{\mu \nu 0} \\
& -\frac{3}{2} \Sigma^{-2}\left(\nabla_{\mu} \Sigma\right)^{2}+\frac{1}{16}\left(D_{\mu} M_{M N}\right)\left(D^{\mu} M^{M N}\right) \\
& +\frac{1}{16 \sqrt{2}} \epsilon^{\mu \nu \rho \lambda \sigma} \xi^{M N} B_{\mu \nu M}\left(D_{\rho} B_{\lambda \sigma N}+8 \eta_{N P} A_{\rho}^{P} \partial_{\lambda} A_{\sigma}^{0}\right) \\
& -\frac{1}{\sqrt{2}} \epsilon^{\mu \nu \rho \lambda \sigma} \eta_{M N} A_{\mu}^{0} \partial_{\nu} A_{\rho}^{M} \partial_{\lambda} A_{\sigma}^{N} \\
& -\frac{1}{16} \xi_{M N} \xi_{P Q} \Sigma^{4}\left(M^{M P} M^{N Q}-\eta^{M P} \eta^{N Q}\right) .
\end{aligned}
$$

In this expression $R$ denotes the Ricci scalar and we define

$$
\mathcal{H}_{\mu \nu}^{M}:=F_{\mu \nu}^{M}-\xi_{N}^{M} A_{[\mu}^{0} A_{\nu]}^{N}+\frac{1}{2} \xi^{M N} B_{\mu \nu N}
$$

where

$$
F_{\mu \nu}^{M}:=\partial_{\mu} A_{\nu}^{M}-\partial_{\nu} A_{\mu}^{M}, \quad F_{\mu \nu}^{0}:=\partial_{\mu} A_{\nu}^{0}-\partial_{\nu} A_{\mu}^{0} .
$$

The vectors are subject to gauge transformations with scalar parameters $\left(\Lambda^{0}, \Lambda^{M}\right)$, while the tensors transform under standard two-form gauge transformations with one-form parameters $\Xi_{\mu M}$. The variation of a vector under these transformations will play a prominent role in the work of this paper, since it allows to implement the tensorial Higgs mechanism. It reads for our choice of gaugings

$$
\delta A_{\mu}^{0}=\nabla_{\mu} \Lambda^{0}, \quad \delta A_{\mu}^{M}=\nabla_{\mu} \Lambda^{M}-A_{\mu}^{0} \xi_{N}{ }^{M} \Lambda^{N}-\frac{1}{2} \xi^{M N} \Xi_{\mu N} .
$$

Next turn to the fermionic Lagrangian. For the purpose of this work it will be sufficient to only recall the kinetic terms, the mass terms as well as further terms quadratic in the fields. A more complete discussion can be found in $[17,18]$. The quadratic fermionic terms of interest read

$$
\begin{aligned}
e^{-1} \mathcal{L}_{\mathrm{ferm}}= & -\frac{1}{2} \bar{\psi}_{\mu}^{i} \gamma^{\mu \nu \rho} \mathcal{D}_{\nu} \psi_{\rho i}-\frac{1}{2} \bar{\chi}^{i} \not \mathcal{D} \chi_{i}-\frac{1}{2} \bar{\lambda}^{i a} \not{D} \lambda_{i}^{a} \\
& +\frac{\sqrt{6} i}{4} A_{1 i j} \bar{\psi}_{\mu}^{i} \gamma^{\mu \nu} \psi_{\nu}^{j}+\left(-\frac{1}{2 \sqrt{2}} \Sigma^{2} \xi_{a b} \Omega_{i j}+\frac{\sqrt{6}}{4} A_{1 i j} \delta_{a b}\right) i \bar{\lambda}^{i a} \lambda^{j b} \\
& -\frac{5 \sqrt{6} i}{12} A_{1 i j} \bar{\chi}^{i} \chi^{j}-\sqrt{2} A_{2 a i j} \bar{\psi}_{\mu}^{i} \gamma^{\mu} \lambda^{j a}-\sqrt{2} A_{1 i j} \bar{\psi}_{\mu}^{i} \gamma^{\mu} \chi^{j}-\frac{4 \sqrt{6} i}{3} A_{2 a i j} \bar{\chi}^{i} \lambda^{j a},
\end{aligned}
$$

where

$$
A_{1}^{i j}=-\frac{\sqrt{3}}{3} \Sigma^{2} \Omega_{k l} \mathcal{V}_{M}{ }^{i k} \mathcal{V}_{N}{ }^{j l} \xi^{M N}, \quad A_{2}^{a i j}=-\frac{1}{2} \Sigma^{2} \mathcal{V}_{M}{ }^{a} \mathcal{V}_{N}{ }^{i j} \xi^{M N}
$$


The first line in (2.21) are the kinetic terms of the fermions, while the remaining two lines summarize their mass terms. The covariant derivatives are given by

$$
\begin{aligned}
\mathcal{D}_{\mu} \psi_{i \nu} & =\nabla_{\mu} \psi_{\nu i}+\frac{\sqrt{3}}{\Sigma^{2}} A_{\mu}^{0} A_{1 i}{ }^{j} \psi_{\nu j}+\ldots, \\
\mathcal{D}_{\mu} \chi_{i} & =\nabla_{\mu} \chi_{i}+\frac{\sqrt{3}}{\Sigma^{2}} A_{\mu}^{0} A_{1 i}{ }^{j} \chi_{j}+\ldots, \\
\mathcal{D}_{\mu} \lambda_{i}^{a} & =\nabla_{\mu} \lambda_{i}^{a}+\frac{\sqrt{3}}{\Sigma^{2}} A_{\mu}^{0} A_{1 i}{ }^{j} \lambda_{j}^{a}+A_{\mu}^{0} \xi^{a}{ }_{b} \lambda_{i}^{b}+\ldots,
\end{aligned}
$$

where the dots indicate couplings to scalars, which are of no importance in this work. In order to simplify expressions like (2.21) we introduce some convenient notation. We denote contractions of the embedding tensor with the coset representatives by

$$
\xi^{m n}:=\mathcal{V}_{M}{ }^{m} \xi^{M N} \mathcal{V}_{N}{ }^{n}, \quad \xi^{a b}:=\mathcal{V}_{M}{ }^{a} \xi^{M N} \mathcal{V}_{N}{ }^{b}, \quad \xi^{a m}:=\mathcal{V}_{M}{ }^{a} \xi^{M N} \mathcal{V}_{N}{ }^{m}
$$

Thus these quantities are field-dependent and only become constant in the vacuum. Note that in the definition of $\xi^{m n}$ and $\xi^{a m}$ the positions of the $\mathrm{SO}(5, n)$-indices $M, N$ are crucial because of (2.10). The local isomorphism of $\mathrm{SO}(5)$ and $\mathrm{USp}(4)$ of (2.13) establishes relations between (2.22) and (2.24). More precisely, one finds using (A.15)

$$
\begin{aligned}
\xi^{m n} & =-\frac{\sqrt{3}}{2 \Sigma^{2}} A_{1}^{i j} \Gamma_{i j}^{m n}, & A_{1}^{i j} & =-\frac{1}{4 \sqrt{3}} \Sigma^{2} \xi^{m n} \Gamma_{m n}{ }^{i j}, \\
\xi^{a m} & =-\frac{1}{\Sigma^{2}} A_{2}^{a i j} \Gamma^{m}{ }_{i j}, & A_{2}^{a i j} & =-\frac{1}{4} \Sigma^{2} \xi^{a m} \Gamma_{m}{ }^{i j},
\end{aligned}
$$

where $\Gamma_{m n}{ }^{i j}$ are the components of the gamma-matrix product $\Gamma_{m n}=\Gamma_{[m} \Gamma_{n]}$.

\subsection{Vacuum conditions and supersymmetry breaking}

In this subsection we formulate the conditions for finding vacua of the gauged $\mathcal{N}=4$ supergravity theory introduced above. We restrict our considerations to the case for which $\xi_{M N}$ is the only non-vanishing embedding tensor such that (2.15) holds. The scalar potential $V$ found in $(2.17)$ is then given by $[17,18]$

$$
V=\frac{1}{16} \xi_{M N} \xi_{P Q} \Sigma^{4}\left(M^{M P} M^{N Q}-\eta^{M P} \eta^{N Q}\right)
$$

Inserting the explicit expressions (2.11) and (2.12) of $\eta_{M N}$ and $M_{M N}$ in terms of the coset representatives gives

$$
V=\frac{1}{4} \xi^{M N} \xi^{P Q} \Sigma^{4} \mathcal{V}_{M}{ }^{a} \mathcal{V}_{P a} \mathcal{V}_{N}{ }^{m} \mathcal{V}_{Q m}=\frac{1}{4} \Sigma^{4} \xi^{a m} \xi_{a m}
$$

where we have inserted (2.24). Using the fact that $a$ and $m$ indices are raised by the Kronecker delta, this implies that the scalar potential is a sum of positive semi-definite terms.

Determining the minima of this potential is now trivial. The derivative with respect to $\Sigma$ yields

$$
\frac{\partial V}{\partial \Sigma}=\Sigma^{3} \xi^{a m} \xi_{a m} \stackrel{!}{=} 0
$$


Since the left-hand-side of this equation is a sum of non-negative quadratic terms, the solution simply reads ${ }^{4}$

$$
\xi^{a m} \stackrel{!}{=} 0 \quad \forall a, m
$$

The potential at this point is

$$
\left.V\right|_{\xi^{a m}=0}=0 .
$$

The remaining derivatives with respect to the scalars in the vector multiplets are trivially vanishing since the potential is quadratic in the $\xi^{a m}$.

In summary, for vanishing embedding tensors $f_{M N P}, \xi_{M}$ the vacua are characterized by the condition $\xi^{a m}=0$ for all $a, m$. Due to (2.30) all such vacua are necessarily Minkowskian. Furthermore, we note that due to the vacuum condition the fermion shift matrix $A_{2}^{a i j}$ given in (2.25) trivially vanishes.

Let us next discuss the amount of supersymmetry preserved by these vacua. It can be determined by analyzing the variations of the fermions under supersymmetry transformations, which can be found in [17]. Evaluated in the vacuum (2.29) they read

$$
\delta \psi_{\mu}^{i}=-\frac{i}{\sqrt{6}} A_{1 i}{ }^{j} \gamma_{\mu} \epsilon_{j}, \quad \delta \chi_{i}=-\sqrt{2} A_{1 i}{ }^{j} \epsilon_{j}, \quad \delta \lambda_{i}^{a}=0,
$$

where $\epsilon_{i}=\epsilon_{i}(x)$ is the supersymmetry parameter. The automatic vanishing of $\delta \lambda_{i}^{a}$ is a direct consequence of the fact that $A_{2}^{a i j}=0$ in the vacuum. It is convenient to decompose the supersymmetry parameter $\epsilon_{i}$ into a Killing spinor $\eta$ and a spacetime independent $\mathrm{USp}(4)$ vector $q_{i}$

$$
\epsilon_{i}=q_{i} \eta .
$$

Each conserved supersymmetry corresponds to a zero eigenvalue of the matrix $A_{1 i}{ }^{j}$. Therefore we now need to face the task of finding the general form of the eigenvalues of $A_{1 i}{ }^{j}$.

The eigenvalues of $A_{1 i}{ }^{j}$ were already determined in [31] and we will recall their analysis in the following. To begin with one considers the decomposition of the $\mathcal{N}=4$ R-symmetry group USp(4) under the choice of a special direction. Recall that there is the local isomorphism $\mathrm{USp}(4) \cong \mathrm{SO}(5)$. One then defines an $\mathrm{SO}(5)$ vector

$$
\tilde{X}^{m}:=\varepsilon^{m n p q r} \xi_{n p} \xi_{q r},
$$

which specifies a preferred direction in $\mathbb{R}^{5}$ and therefore encodes the breaking

$$
\mathrm{SO}(5) \rightarrow \mathrm{SO}(4) \cong \mathrm{SU}(2)_{+} \times \mathrm{SU}(2)_{-} .
$$

Here $\varepsilon^{m n p q r}$ denotes the usual five-dimensional Levi-Civita symbol with $\varepsilon^{12345}=1$. One also defines a unit vector for $\tilde{X} \neq 0$

$$
X^{m}:=\tilde{X}^{m} /|\tilde{X}|
$$

with

$$
|\tilde{X}|=\sqrt{\tilde{X}^{m} \tilde{X}_{m}}=\sqrt{8\left(\xi^{m n} \xi_{m n}\right)^{2}-16 \xi^{m n} \xi_{n p} \xi^{p q} \xi_{q m}} .
$$

\footnotetext{
${ }^{4}$ Let us stress once more that $\xi^{a m}$ is a field-dependent quantity.
} 
Via this construction, the four eigenvalues of the hermitian matrix $i A_{1 i}{ }^{j}$ are given by $\pm a_{1 \pm}$, where [31]

$$
\begin{aligned}
a_{1 \pm} & =\frac{1}{4 \sqrt{3}} \Sigma^{2} \sqrt{2 \xi^{m n} \xi_{m n} \mp|\tilde{X}|} \\
& =\frac{1}{4 \sqrt{3}} \Sigma^{2} \sqrt{2 \xi^{m n} \xi_{m n} \mp \sqrt{8\left(\xi^{m n} \xi_{m n}\right)^{2}-16 \xi^{m n} \xi_{n p} \xi^{p q} \xi_{q m}}}
\end{aligned}
$$

The index of the eigenvalues indicates the corresponding $\mathrm{SU}(2)_{ \pm}$projected subspace. Recall that zero eigenvalues of $A_{1 i}{ }^{j}$ are in one-to-one correspondence with conserved supersymmetries. From (2.37) we see that there are three different possibilities for the amount of supersymmetry in the vacuum: ${ }^{5}$

\begin{tabular}{|c|c|}
\hline supersymmetry & condition \\
\hline $\mathcal{N}=4$ & $\xi^{m n}=0 \quad \forall m, n$ \\
\hline $\mathcal{N}=2$ & $\xi^{m n} \xi_{n p} \xi^{p q} \xi_{q m}=\frac{1}{4}\left(\xi^{m n} \xi_{m n}\right)\left(\xi^{p q} \xi_{p q}\right)$ \\
\hline $\mathcal{N}=0$ & others \\
\hline
\end{tabular}

\section{Supergravity breaking from $\mathcal{N}=4$ to $\mathcal{N}=2$}

In this section we study the supersymmetry breaking from $\mathcal{N}=4$ to $\mathcal{N}=2$. We first comment further on the $\mathcal{N}=2$ vacuum conditions in subsection 3.1. This is followed by a discussion of the spectrum parameterizing fluctuations around this vacuum. More precisely, we focus on the fields that become massive by a Higgs mechanism. In subsection 3.2 we show how some of the tensors become massive by eating vector degrees of freedom, a mechanism that we term tensorial Higgs mechanism. The Higgs mechanism rendering half of the gravitinos massive is summarized in subsection 3.3. Let us stress that both massive tensors and massive gravitinos will play a distinctive role in the evaluation of the one-loop effective action presented in section 4 .

\subsection{Solution to the $\mathcal{N}=2$ condition}

Since we are focusing on $\mathcal{N}=2$ vacua in this work, we recall the corresponding supersymmetry condition from subsection 2.2. It takes the form

$$
\xi^{m n} \xi_{n p} \xi^{p q} \xi_{q m} \stackrel{!}{=} \frac{1}{4}\left(\xi^{m n} \xi_{m n}\right)\left(\xi^{p q} \xi_{p q}\right) \quad \text { and } \quad \exists m, n \text { s.t. } \xi^{m n} \neq 0
$$

where $\xi_{m n}$ is field dependent since it arises from the constant $\xi_{M N}$ via (2.24). In this case the eigenvalues $a_{1 \pm}$ of the gravitino mass matrix given in (2.37) take the form

$$
a_{1+}=0, \quad a_{1-}=\frac{1}{2 \sqrt{3}} \Sigma^{2} \sqrt{\xi^{m n} \xi_{m n}} .
$$

This implies that the unbroken R-symmetry is $\mathrm{SU}(2)_{+}$in $(2.34)$.

\footnotetext{
${ }^{5}$ Note that $\xi^{m n} \xi_{m n}$ is quadratic in each summand.
} 
In the following we aim to find the general solution to (3.1). In order to do that we recall from [31] that $\xi^{m n}$ only acts on the orthogonal complement to $X^{m}$, i.e. it satisfies the vanishing condition

$$
X^{m} \xi_{m n}=0 .
$$

We use the local $\mathrm{SO}(5)$ symmetry in order to rotate

$$
m \rightarrow(\tilde{0}, \hat{m}),
$$

where the index $\tilde{0}$ refers to the direction of $X^{m}$. Then (3.3) ensures that $\xi^{\tilde{0} m}=0$. Note that we still retain the freedom of a reflection along the $X^{m}$ direction as well as $\mathrm{SO}(4) \subset \mathrm{SO}(5)$ rotations orthogonal to $X^{m}$. We continue with the latter in order to bring the skewsymmetric matrix $\xi^{\hat{m} \hat{n}}$ into block-diagonal form [32]

$$
\left(\xi^{m n}\right)=\left(\begin{array}{cc}
0 & 0 \\
0 & \xi^{\hat{m} \hat{n}}
\end{array}\right)=\left(\begin{array}{ccc}
0 & 0 & 0 \\
0 & \gamma \varepsilon & 0 \\
0 & 0 & \tilde{\gamma} \varepsilon
\end{array}\right),
$$

where $\gamma, \tilde{\gamma} \in \mathbb{R}$ and $\varepsilon$ denotes the usual two-dimensional epsilon tensor. The values $\pm i \gamma, \pm i \tilde{\gamma}$ are the imaginary eigenvalues of $\xi^{m n}$. Inserting this expression for $\xi^{m n}$ into (3.1) yields the condition

$$
\gamma \stackrel{!}{=} \pm \tilde{\gamma}
$$

Therefore the $\mathcal{N}=2$ supersymmetry conditions can be summarized as

$$
\mathcal{N}=2 \text { SUSY } \Leftrightarrow \quad\left(\xi^{m n}\right) \text { has eigenvalues } 0, \pm i \gamma, \pm i \gamma \neq 0 .
$$

Note that we can employ the $\mathrm{SO}(5)$ symmetry further to simplify the analysis. In fact, one can always choose

$$
\gamma>0 \quad \text { and } \quad \tilde{\gamma}=\gamma,
$$

which is possible since

$$
\begin{aligned}
\operatorname{diag}(1,-1,1,-1,1) & \in \mathrm{SO}(5), \\
\operatorname{diag}(-1,1,1,-1,1), \operatorname{diag}(-1,-1,1,1,1) & \in \mathrm{SO}(5)
\end{aligned}
$$

are rotations that leave the $\xi^{\tilde{0} m}$ components invariant. The transformation (3.9) changes the absolute signs of $\gamma$ and $\tilde{\gamma}$, while the transformations in (3.10) change the relative sign between $\gamma$ and $\tilde{\gamma}$. Note that (3.9) leaves the direction of $X^{m}$ invariant, while the transformations (3.10) reflect along it.

\subsection{The tensorial Higgs mechanism}

In this subsection we introduce the tensorial Higgs mechanism that is key to implementing the $\mathcal{N}=4$ to $\mathcal{N}=2$ supergravity breaking. In order to do that we recall that the tensors appear in the combination (2.18) as

$$
\mathcal{H}_{\mu \nu}^{M}=F_{\mu \nu}^{M}-\xi_{N}^{M} A_{[\mu}^{0} A_{\nu]}^{N}+\frac{1}{2} \xi^{M N} B_{\mu \nu N} .
$$

In order to perform the Higgs mechanism we apply the gauge transformations (2.20) for the perturbations around the $\mathcal{N}=2$ vacuum. We will show explicitly which vector degrees of freedom get eaten by the tensors to render them massive. 
Since the gauge transformation (2.20) of $A^{0}$ is not dependent on the gauge parameters $\Xi_{\mu N}$ of the tensors, $A^{0}$ will stay massless in the $\mathcal{N}=2$ vacuum. In contrast, some of the $A_{\mu}^{M}$ can be absorbed by the tensors. To make this explicit, we introduce

$$
\begin{aligned}
B_{\mu \nu m} & :=\langle\mathcal{V}\rangle^{M}{ }_{m} B_{\mu \nu M}, & B_{\mu \nu a} & :=\langle\mathcal{V}\rangle^{M}{ }_{a} B_{\mu \nu M}, \\
A_{\mu}^{m} & :=\langle\mathcal{V}\rangle_{M}{ }^{m} A_{\mu}^{M}, & A_{\mu}^{a} & :=\langle\mathcal{V}\rangle_{M}{ }^{a} A_{\mu}^{M},
\end{aligned}
$$

where the $\langle\mathcal{V}\rangle_{M}{ }^{m},\langle\mathcal{V}\rangle_{M}{ }^{a}$ are the vacuum expectation values (VEVs) of the coset representatives $\mathcal{V}_{M}{ }^{m}, \mathcal{V}_{M}{ }^{a}$ in the $\mathcal{N}=2$ vacuum. Note that the position of the indices $M, N$ is crucial in these definitions. Similarly, we can introduce the gauge parameters $\left(\Lambda^{m}, \Lambda^{a}\right)$ and $\left(\Xi_{\mu n}, \Xi_{\mu a}\right)$ by setting

$$
\begin{array}{rlrl}
\Lambda^{m} & :=\Lambda^{M}\langle\mathcal{V}\rangle_{M}{ }^{m}, & \Lambda^{a}: & :=\Lambda^{M}\langle\mathcal{V}\rangle_{M}{ }^{a}, \\
\Xi_{\mu m} & :=\Xi_{\mu M}\langle\mathcal{V}\rangle^{M}{ }_{m}, & \Xi_{\mu a}:=\Xi_{\mu M}\langle\mathcal{V}\rangle^{M}{ }_{a} .
\end{array}
$$

In this rotated basis the gauge transformations (2.20) read

$$
\begin{gathered}
\delta A_{\mu}^{m}=\partial_{\mu} \Lambda^{m}+A_{\mu}^{0} \xi_{p}{ }^{m} \Lambda^{p}-\frac{1}{2} \xi^{m n} \Xi_{\mu n} \\
\delta A_{\mu}^{a}=\partial_{\mu} \Lambda^{a}-A_{\mu}^{0} \xi_{b}{ }^{a} \Lambda^{b}-\frac{1}{2} \xi^{a b} \Xi_{\mu b},
\end{gathered}
$$

where we have inserted the vacuum condition $\xi^{a m}=0$.

In the next step we apply the $\mathrm{SO}(5) \times \mathrm{SO}(n)$ symmetry to rotate $\xi^{m n}$ and $\xi^{a b}$ into a convenient basis. For $\xi^{m n}$ we have done this already in (3.5) and introduced the constant $\gamma$. Similarly, we now split

$$
a \rightarrow(\tilde{a}, \hat{a}), \quad \hat{a}=1, \ldots, \operatorname{rank}\left(\xi^{a b}\right) .
$$

Since $\xi^{a b}$ is anti-symmetric one notes that $\operatorname{rank}\left(\xi^{a b}\right)$ is even and we can define

$$
n_{T}:=\frac{1}{2} \operatorname{rank}\left(\xi^{a b}\right) .
$$

Using the $\mathrm{SO}(n)$ rotations one can then choose a basis such that $\xi^{a b}$ takes the form

$$
\left(\xi^{a b}\right)=\left(\begin{array}{cc}
0 & 0 \\
0 & \xi^{\hat{a} \hat{b}}
\end{array}\right)=\left(\begin{array}{cccc}
0 & 0 & 0 & 0 \\
0 & \gamma_{1} \varepsilon & \cdots & 0 \\
0 & \vdots & \ddots & \vdots \\
0 & 0 & \cdots & \gamma_{n_{T}} \varepsilon
\end{array}\right)
$$

where $\varepsilon$ is the two-dimensional epsilon tensor. In this expression the non-zero real eigenvalues $\gamma_{\breve{a}}, \check{a}=1, \ldots, n_{T}$ are parameterizing the non-trivial VEV of $\xi^{a b}$.

Together (3.5) and (3.18) provide a diagonalization of $\xi^{M N}$ after contraction with the VEVs $\langle\mathcal{V}\rangle$. Recall, however, that the eigenvalues $\pm i \gamma, \pm i \gamma_{\check{a}}$ are identical to the eigenvalues of $\xi^{M N}$, since the contraction with $\langle\mathcal{V}\rangle$ corresponds to a similarity transformation.

Using the explicit expressions (3.5) and (3.18) in the gauge transformations (3.14) and (3.15) we first note that $\delta A_{\mu}^{\tilde{0}}$ and $\delta A_{\mu}^{\tilde{a}}$ simply reduce to

$$
\delta A^{\tilde{0}}=\partial_{\mu} \Lambda^{\tilde{0}}, \quad \delta A_{\mu}^{\tilde{a}}=\partial_{\mu} \Lambda^{\tilde{a}} .
$$


These are the standard gauge transformations for $\mathrm{U}(1)$ gauge fields. We conclude that the vector fields $\left(A^{0}, A^{\tilde{0}}, A^{\tilde{a}}\right)$ remain massless and propagate in the $\mathcal{N}=2$ effective theory describing the massless fluctuations around the vacuum. Turning to the massive degrees of freedom, we can pick the gauge parameters $\left(\Xi_{\mu \hat{n}}, \Xi_{\mu \hat{b}}\right)$ such that

$$
A_{\mu}^{\hat{m}} \stackrel{!}{=} \frac{1}{2} \xi^{\hat{m} \hat{n}} \Xi_{\mu \hat{n}}, \quad A_{\mu}^{\hat{a}} \stackrel{!}{=} \frac{1}{2} \xi^{\hat{a} \hat{b}} \Xi_{\mu \hat{b}}
$$

By definition of $\xi^{\hat{m} \hat{n}}$ and $\xi^{\hat{a} \hat{b}}$ in (3.5) and (3.18) both matrices have full rank and can be inverted to solve for $\left(\Xi_{\mu \hat{n}}, \Xi_{\mu \hat{b}}\right)$. This implies that the vectors $A_{\mu}^{\hat{m}}$ and $A_{\mu}^{\hat{a}}$ are pure gauge and can be absorbed by the tensors $\left(B_{\mu \nu \hat{m}}, B_{\mu \nu \hat{a}}\right)$ to render them massive.

In computing the $\mathcal{N}=2$ effective action for the massless degrees of freedom it will be crucial to include one-loop effects of massive tensors. Therefore it is necessary to evaluate the action of the massive tensors $\left(B_{\mu \nu \hat{m}}, B_{\mu \nu \hat{a}}\right)$ after the gauge-fixing (3.20). Inserting the definitions (2.24), (3.12), and the split form of $\xi^{m n}$ and $\xi^{a b}$ given in the first equalities of (3.5), (3.18) into the general $\mathcal{N}=4$ action (2.17) we extract all terms depending on the tensors. Explicitly, after gauging away the vectors $A_{\mu}^{\hat{m}}, A_{\mu}^{\hat{a}}$, they read

$$
\begin{aligned}
e^{-1} \mathcal{L}_{B}= & \frac{1}{16 \sqrt{2}} \epsilon^{\mu \nu \rho \lambda \sigma}\left\{\xi^{\hat{m} \hat{n}} B_{\mu \nu \hat{m}}\left[\partial_{\rho} B_{\lambda \sigma \hat{n}}+\xi^{\hat{p}} A_{\rho}^{0} B_{\lambda \sigma \hat{p}}\right]\right. \\
& \left.+\xi^{\hat{a} \hat{b}} B_{\mu \nu \hat{a}}\left[\partial_{\rho} B_{\lambda \sigma \hat{b}}-\xi_{\hat{b}}^{\hat{c}} A_{\rho}^{0} B_{\lambda \sigma \hat{c}}\right]\right\} \\
& -\frac{1}{16} \Sigma^{2} \xi^{\hat{m} \hat{p}} \xi_{\hat{m} \hat{q}} B_{\mu \nu \hat{p}} B^{\mu \nu \hat{q}}-\frac{1}{16} \Sigma^{2} \xi^{\hat{\hat{a}} \hat{b}} \xi_{\hat{a} \hat{c}} B_{\mu \nu \hat{b}} B^{\mu \nu \hat{c}} .
\end{aligned}
$$

The first two lines in this expression are the kinetic terms of $\left(B_{\mu \nu \hat{m}}, B_{\mu \nu}\right)$, while the last line summarizes their mass terms. Since $\xi^{\hat{m} \hat{n}}$ and $\xi^{\hat{a} \hat{b}}$ have maximal rank, indeed all such tensors are massive.

The Lagrangian (3.21) can be further simplified by going to the basis in which $\xi^{\hat{m} \hat{n}}, \xi^{\hat{a} \hat{b}}$ are parametrized by the eigenvalues $\gamma, \gamma_{\check{a}}$ as in (3.5), (3.18). The appearance of the twodimensional epsilon tensor in these expressions makes it natural to define the complex tensors

$$
\begin{aligned}
& \boldsymbol{B}_{\alpha}:=B_{2 \alpha-1}+i B_{2 \alpha}, \quad \alpha=1,2 \quad \text { (use } B_{\hat{m}} \text { ) } \\
& \left.\boldsymbol{B}_{\check{a}}:=B_{2 \check{a}-1}+i B_{2 \check{a}}, \quad \check{a}=1, \ldots, n_{T} \quad \text { (use } B_{\hat{a}}\right)
\end{aligned}
$$

with $n_{T}$ defined in (3.17). One can show that the $\alpha$ index corresponds to the fundamental representation of the $\mathcal{N}=2 \mathrm{R}$-symmetry group $\mathrm{SU}(2)$. Here and in the following we will use boldface symbols to denote complex fields. Inserting these definitions together with (3.5) and (3.18) into (3.21) we find ${ }^{6}$

$$
\begin{aligned}
e^{-1} \mathcal{L}_{B}= & -\frac{1}{16}\left[i \frac{1}{\sqrt{2}} \epsilon^{\mu \nu \rho \lambda \sigma} \gamma \overline{\boldsymbol{B}}_{\mu \nu}^{\alpha}\left(\partial_{\rho} \boldsymbol{B}_{\lambda \sigma \alpha}+i \gamma \boldsymbol{B}_{\lambda \sigma \alpha} A_{\rho}^{0}\right)+\Sigma^{2} \gamma^{2} \overline{\boldsymbol{B}}_{\mu \nu}^{\alpha} \boldsymbol{B}_{\alpha}^{\mu \nu}\right] \\
& -\frac{1}{16} \sum_{\check{a}}\left[i \frac{1}{\sqrt{2}} \epsilon^{\mu \nu \rho \lambda \sigma} \gamma_{\breve{a}} \overline{\boldsymbol{B}}_{\mu \nu \check{a}}\left(\partial_{\rho} \boldsymbol{B}_{\lambda \sigma \check{a}}-i \gamma_{\breve{a}} \boldsymbol{B}_{\lambda \sigma \check{a}} A_{\rho}^{0}\right)+\Sigma^{2} \gamma_{\breve{a}}^{2} \overline{\boldsymbol{B}}_{\mu \nu \check{a}} \boldsymbol{B}_{\check{a}}^{\mu \nu}\right] .
\end{aligned}
$$

\footnotetext{
${ }^{6}$ In analogy to the fermions we define $\overline{\boldsymbol{B}}^{\alpha}:=\left(\boldsymbol{B}_{\alpha}\right)^{*}$.
} 


\begin{tabular}{|c|c|c|c|}
\hline & rotation with $\langle\mathcal{V}\rangle$ & $\xi^{M N}$-split & physical degrees \\
\hline \multirow{4}{*}{$\left(A^{M}, B_{M}\right)$} & $\left(A^{m}, B_{m}\right)$ & $\left(A^{\tilde{0}}, B_{\tilde{0}}\right)$ & $A^{\tilde{0}}$ massless \\
\cline { 3 - 4 } & & $\left(A^{\hat{m}}, B_{\hat{m}}\right)$ & $\boldsymbol{B}_{\alpha}$ complex, massive \\
\cline { 2 - 4 } & \multirow{2}{*}{$\left(A^{a}, B_{a}\right)$} & $\left(A^{\tilde{a}}, B_{\tilde{a}}\right)$ & $A^{\tilde{a}}$ massless \\
\cline { 3 - 4 } & $\left(A^{\hat{a}}, B_{\hat{a}}\right)$ & $\boldsymbol{B}_{\tilde{a}}$ complex, massive \\
\hline
\end{tabular}

Table 1. Natural split of $A^{M}$ and $B_{M}$ induced by $\xi^{M N}$.

In the last step we want to rescale the complex tensors in order to bring the action into the standard form

$$
e^{-1} \mathcal{L}_{B}=-\frac{1}{4} i c_{\boldsymbol{B}} \epsilon^{\mu \nu \rho \sigma \tau} \overline{\boldsymbol{B}}_{\mu \nu} \mathcal{D}_{\rho} \boldsymbol{B}_{\sigma \tau}-\frac{1}{2} m_{\boldsymbol{B}} \overline{\boldsymbol{B}}_{\mu \nu} \boldsymbol{B}^{\mu \nu},
$$

with $\mathcal{D}_{\rho} \boldsymbol{B}_{\sigma \tau}=\partial_{\rho} \boldsymbol{B}_{\sigma \tau}-i q_{\boldsymbol{B}} A_{\rho}^{0} \boldsymbol{B}_{\sigma \tau}$. This Lagrangian was used in the one-loop computations of $[19,20]$. Here $m_{\boldsymbol{B}}>0$ is the real mass of the complex tensor $\boldsymbol{B}_{\mu \nu}$ and $q_{\boldsymbol{B}}$ encodes its charge under the $\mathrm{U}(1)$ vector $A^{0}$. The choice of $c_{\boldsymbol{B}}$ is dependent on the representation of $\boldsymbol{B}_{\mu \nu}$ under the massive little group $\mathrm{SO}(4)$ in five dimensions. Explicitly, one has

$$
\begin{aligned}
& c_{\boldsymbol{B}}=+1 \Leftrightarrow(1,0) \text { of } \mathrm{SO}(4) \\
& c_{\boldsymbol{B}}=-1 \Leftrightarrow(0,1) \text { of } \mathrm{SO}(4) .
\end{aligned}
$$

Comparing (3.23) with (3.24) we can determine $c_{\boldsymbol{B}}, m_{\boldsymbol{B}}, q_{\boldsymbol{B}}$ after rescaling

$$
\left(\boldsymbol{B}_{\alpha}, \boldsymbol{B}_{\check{a}}\right) \mapsto \frac{1}{2^{5 / 4}}\left(\sqrt{\gamma} \boldsymbol{B}_{\alpha}, \sqrt{\left|\gamma_{\check{a}}\right|} \boldsymbol{B}_{\check{a}}\right)
$$

This results in the identifications

$$
\begin{aligned}
& c_{\boldsymbol{B}_{\alpha}}=1, \quad m_{\boldsymbol{B}_{\alpha}}=\frac{1}{\sqrt{2}} \Sigma^{2} \gamma, \quad q_{\boldsymbol{B}_{\alpha}}=-\gamma, \\
& c_{\boldsymbol{B}_{\check{a}}}=\operatorname{sign} \gamma_{\check{a}}, \quad m_{\boldsymbol{B}_{\check{a}}}=\frac{1}{\sqrt{2}} \Sigma^{2}\left|\gamma_{\breve{a}}\right|, \quad q_{\boldsymbol{B}_{\check{a}}}=\gamma_{\check{a}} .
\end{aligned}
$$

This concludes our discussion of the massive tensors. We found that evaluated around the $\mathcal{N}=2$ vacuum there are $n_{T}+2$ complex massive tensors $\left(\boldsymbol{B}_{\alpha}, \boldsymbol{B}_{\breve{a}}\right)$ with standard action (3.24) and characteristic data (3.27). For convenience we summarize the split of the fields induced by $\xi^{M N}$ in table 1 .

\subsection{The super-Higgs mechanism}

In the $\mathcal{N}=2$ broken phase of a $\mathcal{N}=4$ theory a gravitino mass term has to be generated for half of the gravitino degrees of freedom. This mass arises in the sector of the broken $\mathrm{SU}(2)$ subgroup of the $\mathcal{N}=4 \mathrm{R}$-symmetry group $\operatorname{USp}(4)$. In fact, two gravitini eat up two spin-1/2 goldstini from the gravity multiplet and become massive. In this super-Higgs mechanism the massive gravitini acquire four extra degrees of freedom. The appropriate description of the massive fields is in terms of a single Dirac spin-3/2 fermion $\boldsymbol{\psi}_{\mu}$ without 
a symplectic Majorana condition. The massive gravitino combines with the two massive complex tensors $\boldsymbol{B}_{\alpha}$ of the gravity multiplet into a massive $\mathcal{N}=2$ gravitino multiplet $\left(\boldsymbol{\psi}_{\mu}, \boldsymbol{B}_{\alpha}\right)$. The construction of such a half-BPS multiplet has been discussed in [33]. In the following we will briefly discuss the super-Higgs mechanism and determine the mass and $\mathrm{U}(1)$ charge of the gravitino multiplet.

Let us first consider the four $\mathcal{N}=4$ symplectic Majorana gravitini $\psi_{\mu}^{i}$ and the spin- $1 / 2$ fermions in the gravity multiplet $\chi^{i}$. These split under the breaking

$$
\mathrm{USp}(4) \rightarrow \mathrm{SU}(2)_{+} \times \mathrm{SU}(2)_{-},
$$

into $\psi_{\mu}^{\alpha}, \psi_{\mu}^{\dot{\alpha}}$ and $\chi^{\alpha}, \chi^{\dot{\alpha}}$, respectively. As noted in the last subsection, the index $\alpha=1,2$ refers to the fundamental representation of the $\mathcal{N}=2$ R-symmetry group $\mathrm{SU}(2)_{+}$, while $\dot{\alpha}=1,2$ corresponds to the broken $\mathrm{SU}(2)_{-}$part. Both indices are raised and lowered with the epsilon tensor analogous to (2.2) and (2.3). As one can see in (2.21) using the $\mathcal{N}=2$ vacuum conditions (2.29) and (3.1) all fermion bilinears involving $\psi_{\mu}^{\alpha}$ and $\chi^{\alpha}$ vanish in the vacuum, leaving only the kinetic terms for these fields. Thus we find two massless $\mathcal{N}=2$ spin-3/2 symplectic Majorana fermions $\psi_{\mu}^{\alpha}$ and two massless spin-1/2 symplectic Majorana fermions $\chi^{\alpha}$. The reduced symplectic Majorana condition reads

$$
\bar{\chi}^{\alpha}:=\left(\chi_{\alpha}\right)^{\dagger} \gamma_{0}=\epsilon^{\alpha \beta} \chi_{\beta}^{T} C
$$

and similarly for $\psi_{\mu}^{\alpha}$, where $C$ denotes the charge conjugation matrix and $\epsilon^{\alpha \beta}$ is the twodimensional Levi-Civita symbol with $\epsilon^{12}=+1$. We note that throughout this paper all massless fermionic $\mathcal{N}=2$ fields are taken to be symplectic Majorana.

We proceed with the investigation of the remaining fields $\psi_{\mu}^{\dot{\alpha}}$ and $\chi^{\dot{\alpha}}$. The terms in the Lagrangian involving these fields are (ignoring fluctuations of scalars)

$$
\begin{aligned}
e^{-1} \mathcal{L}_{\psi, \chi}= & -\frac{1}{2} \bar{\psi}_{\mu}^{\dot{\alpha}} \gamma^{\mu \nu \rho} \mathcal{D}_{\nu} \psi_{\rho \dot{\alpha}}-\frac{1}{2} \bar{\chi}^{\dot{\alpha}} \not \chi_{\chi_{\dot{\alpha}}}+\frac{\sqrt{6} i}{4}\left\langle A_{1}\right\rangle_{\dot{\alpha} \dot{\beta}} \bar{\psi}_{\mu}^{\dot{\alpha}} \gamma^{\mu \nu} \psi_{\nu}^{\dot{\beta}} \\
& -\frac{5 \sqrt{6} i}{12}\left\langle A_{1}\right\rangle_{\dot{\alpha} \dot{\beta}} \bar{\chi}^{\dot{\alpha}} \chi^{\dot{\beta}}-\sqrt{2}\left\langle A_{1}\right\rangle_{\dot{\alpha} \dot{\beta}} \bar{\psi}_{\mu}^{\dot{\alpha}} \gamma^{\mu} \chi^{\dot{\beta}} \\
= & -\frac{1}{2} \bar{\psi}_{\mu}^{\dot{\alpha}} \gamma^{\mu \nu \rho} \mathcal{D}_{\nu} \psi_{\rho \dot{\alpha}}-\frac{1}{2} \bar{\chi}^{\dot{\alpha}} \mathcal{D}_{\chi_{\dot{\alpha}}}-\frac{1}{2 \sqrt{2}} \Sigma^{2} \gamma \bar{\psi}_{\mu}^{\dot{\alpha}} \gamma^{\mu \nu} \psi_{\nu \dot{\alpha}} \\
& +\frac{5 \sqrt{2}}{12} \Sigma^{2} \gamma \bar{\chi}^{\dot{\alpha}} \chi_{\dot{\alpha}}+\frac{\sqrt{6}}{3} \Sigma^{2} \gamma \bar{\psi}_{\mu}^{\dot{\alpha}} \gamma^{\mu} \chi_{\dot{\alpha}},
\end{aligned}
$$

where we used that due to (3.2) the two eigenvalues $\pm a_{1-}$ of $\left\langle A_{1}\right\rangle_{\dot{\alpha}}{ }^{\dot{\beta}}$ are given by

$$
a_{1-}=\frac{1}{\sqrt{3}} \Sigma^{2} \gamma
$$

The $\chi^{\dot{\alpha}}$ actually are the goldstini, that render the $\psi_{\mu}^{\dot{\alpha}}$ massive, and can be removed from the action by a shift of the gravitini analog to the one performed in [15, 34]. We thus obtain

$$
e^{-1} \mathcal{L}_{\text {mass grav }}=-\frac{1}{2} \bar{\psi}_{\mu}^{\dot{\alpha}} \gamma^{\mu \nu \rho} \mathcal{D}_{\nu} \psi_{\dot{\alpha} \rho}-\frac{1}{2} \frac{1}{\sqrt{2}} \Sigma^{2} \gamma \bar{\psi}_{\mu}^{\dot{\alpha}} \gamma^{\mu \nu} \psi_{\dot{\alpha} \nu}
$$


It is now convenient to merge the two symplectic Majorana fermions into a single unconstrained Dirac spinor ${ }^{7}$

$$
\psi_{\mu}:=\psi_{\mu}^{\dot{\alpha}=1}
$$

The action then reads

$$
e^{-1} \mathcal{L}_{\text {mass grav }}=-\overline{\boldsymbol{\psi}}_{\mu} \gamma^{\mu \nu \rho} \mathcal{D}_{\nu} \boldsymbol{\psi}_{\rho}-\frac{1}{\sqrt{2}} \Sigma^{2} \gamma \overline{\boldsymbol{\psi}}_{\mu} \gamma^{\mu \nu} \boldsymbol{\psi}_{\nu}
$$

with $\mathcal{D}_{\mu} \boldsymbol{\psi}_{\nu}=\partial_{\mu} \boldsymbol{\psi}_{\nu}-i \gamma A_{\mu}^{0} \boldsymbol{\psi}_{\nu}$

To conclude this section we compare the action (3.34) with the standard form

$$
e^{-1} \mathcal{L}_{\psi}=-\overline{\boldsymbol{\psi}}_{\mu} \gamma^{\mu \nu \rho} \mathcal{D}_{\nu} \boldsymbol{\psi}_{\rho}-c_{\boldsymbol{\psi}} m_{\boldsymbol{\psi}} \overline{\boldsymbol{\psi}}_{\mu} \gamma^{\mu \nu} \boldsymbol{\psi}_{\nu}
$$

where $\mathcal{D}_{\nu} \boldsymbol{\psi}_{\rho}=\partial_{\nu} \boldsymbol{\psi}_{\rho}-i q_{\boldsymbol{\psi}} A_{\nu} \boldsymbol{\psi}_{\rho}$ and $c_{\boldsymbol{\psi}}$ depends on the representation under the massive little group $\mathrm{SO}(4)$ as

$$
\begin{aligned}
& c_{\psi}=+1 \Leftrightarrow\left(\frac{1}{2}, 1\right) \text { of } \mathrm{SO}(4), \\
& c_{\psi}=-1 \Leftrightarrow\left(1, \frac{1}{2}\right) \text { of } \mathrm{SO}(4) .
\end{aligned}
$$

Comparing (3.34) with (3.35) we conclude that $\boldsymbol{\psi}$ is in the $\left(\frac{1}{2}, 1\right)$ representation of $\mathrm{SO}(4)$ and carries mass and $A_{\mu}^{0}$-charge

$$
c_{\psi}=1, \quad m_{\psi}=\frac{1}{\sqrt{2}} \Sigma^{2} \gamma, \quad q_{\psi}=\gamma .
$$

These data will be crucial in evaluating the one-loop corrections induced by the massive gravitino multiplet in the next section.

The massive Dirac gravitino $\boldsymbol{\psi}$ combines with the massive tensors $\boldsymbol{B}_{\alpha}$ of the last section into a massive gravitino multiplet.

\section{Mass spectrum and effective action}

In this section we identify the complete spectrum parameterizing the fluctuations around the $\mathcal{N}=2$ vacuum. We determine the masses and $\mathrm{U}(1)$ charges of all fields and show how they reassemble in $\mathcal{N}=2$ multiplets in subsection 4.1. Furthermore, we derive the low-energy effective action of the massless modes with particular focus on the data determining the $\mathcal{N}=2$ vector sector. The classical truncation from $\mathcal{N}=4$ to $\mathcal{N}=2$ is discussed in subsection 4.2. The crucial inclusion of one-loop quantum corrections due to integrating out massive fermions and tensors is discussed in subsection 4.3. These induce extra contributions to the metric and Chern-Simons terms that are independent of the scale of supersymmetry breaking.

\footnotetext{
${ }^{7}$ We could also choose $\boldsymbol{\psi}_{\mu}:=\psi_{\mu}^{\dot{\alpha}=2}$, which flips the representation and the charge under $A_{\mu}^{0}$, since both descriptions are equivalent.
} 


\subsection{The $\mathcal{N}=2$ spectrum}

The $\mathcal{N}=2$ spectrum and its properties can be determined by evaluating the $\mathcal{N}=4$ action in the vicinity of the $\mathcal{N}=2$ vacuum. To read off the masses and charges all kinetic terms and mass terms have to be brought into canonical form after spontaneous symmetry breaking. This diagonalization procedure is rather lengthy and therefore partially deferred to appendix B in detail. In the following we highlight some of the basic steps and summarize the results.

The key ingredients in the mass generation are the gaugings $\xi^{M N}$. Recall that in the background we rotated $\xi^{M N}$ to $\xi^{m n}, \xi^{a b}$ and found the components

$$
\begin{aligned}
\xi^{m n} \rightarrow \xi^{\hat{m} \hat{n}}, & \xi^{\tilde{0} \hat{n}}=\xi^{\tilde{0} \tilde{0}}=0, \\
\xi^{a b} \rightarrow \xi^{\hat{a} \hat{b}}, & \xi^{\tilde{a} \hat{b}}=\xi^{\tilde{a} \tilde{b}}=0,
\end{aligned}
$$

where $\xi^{\hat{m} \hat{n}}$ and $\xi^{\hat{a} \hat{b}}$ have maximal rank. This yielded the natural index split

$$
\begin{aligned}
m & \rightarrow(\tilde{0}, \hat{m}) \rightarrow(\tilde{0},[\alpha 1],[\alpha 2]), \\
a & \rightarrow(\tilde{a}, \hat{a}) \rightarrow(\tilde{a},[\check{a} 1],[\check{a} 2]) .
\end{aligned}
$$

Here the splits of $\hat{m}$ into $[\alpha 1],[\alpha 2]$ arises due to the block-diagonalization in (3.5) with the first index $\alpha$ labeling the two blocks and the second index labeling the two entries of each

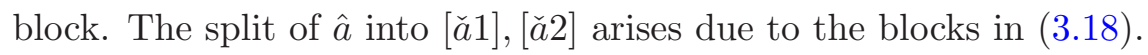

In order to extract the massless and massive scalar spectrum it is convenient to reparameterize the element $\mathcal{V}$ of the coset space $\mathcal{M}_{5, n}$ introduced in (2.7). Denoting by $\langle\mathcal{V}\rangle$ the value of $\mathcal{V}$ in the $\mathcal{N}=2$ vacuum as in (3.12), we write

$$
\mathcal{V}=\langle\mathcal{V}\rangle \exp \left(\phi^{m a}\left[t_{m a}\right]\right)
$$

where $\left[t_{m a}\right]_{M}^{N}=2 \delta_{[m}^{N} \eta_{a] M}$. The $\phi^{m a}$ then correspond to the unconstrained fluctuations around the vacuum value $\langle\mathcal{V}\rangle$ and constitute the scalar degrees of freedom in the $\mathcal{N}=2$ effective theory. Due to the index split (4.3) we need to consider the scalars

$$
\phi^{m a} \rightarrow\left(\phi^{\tilde{0} \tilde{a}}, \phi^{\tilde{0}[\check{a} 1]}, \phi^{\tilde{0}[\check{a} 2]}\right)\left(\phi^{[\alpha 1] \tilde{a}}, \phi^{[\alpha 1][\check{a} 1]}, \phi^{[\alpha 1][\check{a} 2]}\right)\left(\phi^{[\alpha 2] \tilde{a}}, \phi^{[\alpha 2][\check{a} 1]}, \phi^{[\alpha 2][\check{a} 2]}\right) .
$$

To treat these more compactly we introduce, just as for tensors in (3.22), the complex scalars

$$
\begin{aligned}
\phi^{\alpha \tilde{a}} & :=\frac{1}{\sqrt{2}}\left(\phi^{[\alpha 1] \tilde{a}}+i \phi^{[\alpha 2] \tilde{a}}\right), \quad \phi^{\tilde{0} \hat{a}}:=\frac{1}{\sqrt{2}}\left(\phi^{\tilde{0}[\check{a} 1]}+i \phi^{\tilde{0}[\check{a} 2]}\right), \\
\phi_{1}^{\alpha \check{a}}: & =\frac{1}{2}\left(\phi^{[\alpha 1][\check{a} 1]}-\phi^{[\alpha 2][\check{a} 2]}+i \phi^{[\alpha 2][\check{a} 1]}+i \phi^{[\alpha 1][\tilde{a} 2]}\right) \\
\phi_{2}^{\alpha \check{a}} & :=\frac{1}{2}\left(\phi^{[\alpha 1][\check{a} 2]}-\phi^{[\alpha 2][\check{a} 1]}+i \phi^{[\alpha 2][\check{a} 2]}+i \phi^{[\alpha 1][\check{a} 1]}\right) .
\end{aligned}
$$

Note that in this way all $\phi^{m a}$ of the split (4.5) except $\phi^{\tilde{0} \tilde{a}}$ are combined into complex scalars. 
Similarly, we proceed for the split of the $\mathcal{N}=4$ fermions $\lambda_{i}^{a}$. Note that as for the gravitino in subsection 3.3 one splits $i \rightarrow(\alpha, \dot{\alpha})$. Together with the index split of $a$ given in (4.3) one has

$$
\lambda_{i}^{a} \rightarrow\left(\lambda_{\alpha}^{\tilde{a}}, \lambda_{\alpha}^{[\check{a} 1]}, \lambda_{\alpha}^{[\check{a} 2]}\right)\left(\lambda_{\dot{\alpha}}^{\tilde{a}}, \lambda_{\dot{\alpha}}^{[\check{a} 1]}, \lambda_{\dot{\alpha}}^{[\check{a} 2]}\right) .
$$

It turns out to be convenient to combine all $\lambda_{i}^{a}$ except $\lambda_{\alpha}^{\tilde{a}}$ into complex Dirac fermions

$$
\begin{aligned}
& \lambda_{\alpha}^{\check{a}}:=\frac{1}{\sqrt{2}}\left(\lambda_{\alpha}^{[\check{a} 1]}+i \lambda_{\alpha}^{[\check{a} 2]}\right), \\
& \lambda^{\tilde{a}}:=\lambda_{\dot{\alpha}=1}^{\tilde{a}}, \quad \lambda_{1}^{\check{a}}:=\frac{1}{\sqrt{2}}\left(\lambda_{\dot{\alpha}=1}^{[\check{a} 1]}+i \lambda_{\dot{\alpha}=1}^{[\check{a} 2]}\right), \quad \lambda_{2}^{\check{a}}:=\frac{1}{\sqrt{2}}\left(\lambda_{\dot{\alpha}=1}^{[\check{a} 1]}-i \lambda_{\dot{\alpha}=1}^{[\check{a} 2]}\right) .
\end{aligned}
$$

To justify the use of (4.8) we stress that the appearance of all spin- $1 / 2$ fermions can be expressed in terms of the unconstrained Dirac spinors $\boldsymbol{\lambda}_{\alpha}^{\check{a}}, \boldsymbol{\lambda}^{\tilde{a}}, \boldsymbol{\lambda}_{1,2}^{\check{a}}$. Concerning (4.8a), the other linear combination $\frac{1}{\sqrt{2}}\left(\lambda_{\alpha}^{[\check{a} 1]}-i \lambda_{\alpha}^{[\check{a} 2]}\right)$ is related to $\lambda_{\alpha}^{\check{a}}$ by the symplectic Majorana condition. In (4.8b), by the same reasoning, the linear combinations with $\lambda_{\dot{\alpha}=2}^{a}$ are related to those involving $\lambda_{\dot{\alpha}=1}^{a}$. All degrees of freedom of the massive spin- $1 / 2$ fermions are therefore captured by the spinors (4.8), dropping the symplectic Majorana condition.

We are now in the position to summarize the spectrum. From the $\mathcal{N}=4$ gravity multiplet the metric $g_{\mu \nu}$, two gravitini $\psi_{\mu}^{\alpha}$, two spin-1/2 fermions $\chi_{\alpha}$, two vectors $A^{0}, A^{\tilde{0}}$, and one scalar $\Sigma$ remain massless. These fields group into the $\mathcal{N}=2$ gravity multiplet $\left(g_{\mu \nu}, A^{\tilde{0}}, \psi_{\mu}^{\alpha}\right)$ and one $\mathcal{N}=2$ vector multiplet $\left(A^{0}, \Sigma, \chi_{\alpha}\right)$. Note that the vector multiplet $\left(A^{0}, \Sigma, \chi_{\alpha}\right)$ is special, since the massive states, such as the tensors and gravitini discussed in subsection 3.2 and subsection 3.3 , carry $A^{0}$ charge. In order to later derive the quantum effective action for the $A^{0}$ vector multiplet we need to determine the $A^{0}$-charge of all massive states. Additional We stress that the identifications in (4.8b) are analogous to the definition of the massive gravitino (3.33). $n-2 n_{T}$ vector multiplets $\left(A^{\tilde{a}}, \phi^{\tilde{0} \tilde{a}}, \lambda_{\alpha}^{\tilde{a}}\right)$ remain massless. We have already discussed the massless vectors $A^{\tilde{a}}$ in subsection 3.2. Inserting (4.4) into the $\mathcal{N}=4$ action we check in appendix B that the $\phi^{\tilde{0} \tilde{a}}$ and $\lambda_{\alpha}^{\tilde{a}}$ are indeed massless scalars.

Recall that an $\mathcal{N}=2$ hypermultiplet has four real scalars and one Dirac spin- $1 / 2$ fermion. Using the above definitions (4.6) and (4.8) one can form the hypermultiplets

$$
\left(\phi^{\alpha \tilde{a}}, \lambda^{\tilde{a}}\right), \quad\left(\phi_{1}^{\alpha \check{a}}, \lambda_{1}^{\check{a}}\right), \quad\left(\phi_{2}^{\alpha \check{a}}, \lambda_{2}^{\check{a}}\right) .
$$

The $n-2 n_{T}$ hypermultiplets $\left(\phi^{\alpha \tilde{a}}, \boldsymbol{\lambda}^{\tilde{a}}\right)$ are always massive, since they receive masses $m_{\tilde{a}}=\frac{1}{\sqrt{2}} \Sigma^{2} \gamma$ from a non-trivial $\xi^{\hat{m} \hat{n}}$. The hypermultiplets $\left(\phi_{1,2}^{\alpha \check{a}}, \boldsymbol{\lambda}_{1,2}^{\breve{a}}\right)$ can be either massless or massive, since their masses have two contributions from a non-trivial $\xi^{\hat{m} \hat{n}}$ and $\xi^{\hat{a} \hat{b}}$, respectively. As we show in appendix B the $\xi^{M N}$-splits (3.5) and (3.18) yield masses given by

$$
m_{\check{a}}^{1}=\frac{1}{\sqrt{2}} \Sigma^{2}\left|\gamma-\gamma_{\breve{a}}\right|, \quad m_{\check{a}}^{2}=\frac{1}{\sqrt{2}} \Sigma^{2}\left|\gamma+\gamma_{\check{a}}\right|,
$$

for the fields $\left(\phi_{1}^{\alpha \check{a}}, \lambda_{1}^{\check{a}}\right)$ and $\left(\phi_{2}^{\alpha \check{a}}, \boldsymbol{\lambda}_{2}^{\check{a}}\right)$, respectively. This implies that one hypermultiplet is massless whenever the condition

$$
\gamma_{\check{a}}=\gamma \quad \text { or } \quad \gamma_{\check{a}}=-\gamma
$$


is satisfied. We denote the number of such massless hypermultiplets by $n_{H}$, and name them $\left(h_{1,2,3,4}^{\Lambda}, \lambda_{\alpha}^{\Lambda}\right)$, with $\Lambda=1, \ldots, n_{H}$. Due to the fact that the hypermultiplets appear in pairs, the existence of a massless hypermultiplet implies the existence of a massive hypermultiplet with mass $\sqrt{2} \Sigma^{2} \gamma$. Furthermore, one can check that one can consistently choose all $\gamma_{\check{a}}>0$ without changing the effective theory. In summary, one has $2 n_{T}-n_{H}$ massive hypermultiplets with mass (4.10) out of the set $\left(\phi_{1,2}^{\alpha \check{a}}, \boldsymbol{\lambda}_{1,2}^{\check{a}}\right)$. Together with the $\left(\phi^{\alpha \tilde{a}}, \lambda^{\tilde{a}}\right)$ one finds a total of $n-n_{H}$ massive hypermultiplets.

To complete the summary of the spectrum recall that in subsection 3.2 and subsection 3.3 we have already identified and analyzed the $\mathcal{N}=2$ massive gravitino multiplet comprising a massive Dirac gravitino $\boldsymbol{\psi}_{\mu}$ and two complex massive tensors $\boldsymbol{B}_{\alpha}$. Furthermore, we found $n_{T}$ complex massive tensors $\boldsymbol{B}_{\breve{a}}$. The latter combine with Dirac fermions $\boldsymbol{\lambda}_{\alpha}^{\breve{a}}$ into $n_{T}$ complex massive tensor multiplets.

To conclude we list in table 2 the decompositions of the $\mathcal{N}=4$ fields in terms of $\mathcal{N}=2$ fields along with their masses and charges. The reorganization into $\mathcal{N}=2$ multiplets can be found in table 3 .

\subsection{General $\mathcal{N}=2$ action and classical matching}

We are now in the position to derive the classical $\mathcal{N}=2$ effective action for the massless modes. In order to do that we simply truncate the $\mathcal{N}=4$ action to the massless sector. The discussion of the quantum corrections can be found in the next subsection.

To begin with we recall the canonical form of a general $\mathcal{N}=2$ ungauged supergravity theory. The dynamics of the gravity-vector sector is entirely specified in terms of a cubic potential

$$
\mathcal{N}=\frac{1}{3 !} k_{I J K} M^{I} M^{J} M^{K}
$$

where $M^{I}, I=1, \ldots, n-2 n_{T}+2$ are very special real coordinates and $k_{I J K}$ is a symmetric tensor. The $M^{I}$ naturally combine with the vectors $A^{I}$ of the theory. However, since the vector in the gravity multiplet is not accompanied by a scalar degree of freedom, the $M^{I}$ have to satisfy one constraint. In fact, the $\mathcal{N}=2$ scalar field space is identified with the hypersurface

$$
\mathcal{N} \stackrel{!}{=} 1
$$

The gauge coupling function and the metric are then obtained as

$$
G_{I J}=\left[-\frac{1}{2} \partial_{M^{I}} \partial_{M^{J}} \log \mathcal{N}\right]_{\mathcal{N}=1}
$$

The bosonic two-derivative Lagrangian is then given by

$$
\begin{aligned}
\mathcal{L}_{\text {can }}= & -\frac{1}{2} R-\frac{1}{2} G_{I J} \partial_{\mu} M^{I} \partial^{\mu} M^{J}-\frac{1}{4} G_{I J} F_{\mu \nu}^{I} F^{\mu \nu J} \\
& +\frac{1}{48} \epsilon^{\mu \nu \rho \sigma \lambda} k_{I J K} A_{\mu}^{I} F_{\nu \rho}^{J} F_{\sigma \lambda}^{K}-H_{u v} \partial_{\mu} h^{u} \partial^{\mu} h^{v} .
\end{aligned}
$$

Here we included the kinetic term for the hypermultiplet scalars $h^{u}$ with metric $H_{u v}$.

The canonical Lagrangian (4.15) has to be compared with the truncated $\mathcal{N}=4$ theory. In our set-up we found the vectors $\left(A^{I}\right)=\left(A^{0}, A^{\tilde{0}}, A^{\tilde{a}}\right)$, which sets the index range for $I$. 


\begin{tabular}{|c|c|c|c|c|}
\hline $\begin{array}{l}\mathcal{N}=4 \\
\text { fields }\end{array}$ & $\begin{array}{c}\mathcal{N}=2 \\
\text { fields }\end{array}$ & mass & $\begin{array}{l}c_{\text {field }}, \mathrm{SO}(4) \text { rep } \\
\text { for massive fields }\end{array}$ & $\begin{array}{l}\text { charge } \\
\text { under } A^{0}\end{array}$ \\
\hline$g_{\mu \nu}$ & $g_{\mu \nu}$ & 0 & - & 0 \\
\hline$A_{\mu}^{0}$ & $A_{\mu}^{0}$ & 0 & - & 0 \\
\hline$A_{\mu}^{i j}$ & $\begin{array}{c}A_{\mu}^{\tilde{0}} \\
\boldsymbol{B}_{\mu \nu \alpha}\end{array}$ & $\begin{array}{l}0 \\
\frac{1}{\sqrt{2}} \Sigma^{2} \gamma\end{array}$ & $\begin{array}{l}- \\
-1\end{array}$ & $\begin{array}{l}0 \\
\gamma\end{array}$ \\
\hline$\psi_{\mu}^{i}$ & $\begin{array}{l}\psi_{\mu}^{\alpha} \\
\psi_{\mu}\end{array}$ & $\begin{array}{l}0 \\
\frac{1}{\sqrt{2}} \Sigma^{2} \gamma\end{array}$ & $\begin{array}{l}- \\
1\end{array}$ & $\begin{array}{l}0 \\
\gamma\end{array}$ \\
\hline$\chi_{i}$ & $\begin{array}{l}\chi_{\alpha} \\
\chi_{\dot{\alpha}}\end{array}$ & $\begin{array}{l}0 \\
-\end{array}$ & $\begin{array}{l}- \\
\text { goldstino }\end{array}$ & $\begin{array}{l}0 \\
-\end{array}$ \\
\hline$\Sigma$ & $\Sigma$ & 0 & - & 0 \\
\hline$A_{\mu}^{a}$ & $\begin{array}{c}A_{\mu}^{\tilde{a}} \\
\boldsymbol{B}_{\mu \nu \check{a}}\end{array}$ & $\begin{array}{l}0 \\
\frac{1}{\sqrt{2}} \Sigma^{2}\left|\gamma_{\check{a}}\right|\end{array}$ & - & $\begin{array}{l}0 \\
\gamma_{\check{a}} \\
\end{array}$ \\
\hline$\lambda_{i}^{a}$ & $\begin{array}{l}\lambda_{\alpha}^{\tilde{a}} \\
\lambda_{\alpha}^{\check{a}} \\
\lambda^{\tilde{a}} \\
\lambda_{1,2}^{\check{a}} \\
\end{array}$ & $\begin{array}{l}0 \\
\frac{1}{\sqrt{2}} \Sigma^{2}\left|\gamma_{\breve{a}}\right| \\
\frac{1}{\sqrt{2}} \Sigma^{2} \gamma \\
\frac{1}{\sqrt{2}} \Sigma^{2}\left|\gamma \mp \gamma_{\breve{a}}\right|\end{array}$ & $\begin{array}{l}- \\
\operatorname{sign}\left(\gamma_{\check{a}}\right) \\
-1 \\
\operatorname{sign}\left(\gamma \mp \gamma_{\check{a}}\right)\end{array}$ & $\begin{array}{l}0 \\
\gamma_{\check{a}} \\
\gamma \\
\gamma \mp \gamma_{\check{a}}\end{array}$ \\
\hline$\phi^{m a}$ & $\begin{array}{l}\phi^{\tilde{0} \tilde{a}} \\
\phi^{\alpha \tilde{a}} \\
\phi^{\tilde{0} \check{a}} \\
\phi_{1,2}^{\alpha \check{a}}\end{array}$ & $\begin{array}{l}0 \\
\frac{1}{\sqrt{2}} \Sigma^{2} \gamma \\
\frac{1}{\sqrt{2}} \Sigma^{2}\left|\gamma_{\check{a}}\right| \\
\frac{1}{\sqrt{2}} \Sigma^{2}\left|\gamma \mp \gamma_{\check{a}}\right|\end{array}$ & $\begin{array}{l}- \\
\text { singlet } \\
\text { singlet } \\
\text { singlet }\end{array}$ & $\begin{array}{l}0 \\
\gamma \\
\gamma_{\breve{a}} \\
\gamma \mp \gamma_{\check{a}}\end{array}$ \\
\hline
\end{tabular}

Table 2. Decomposition of the $\mathcal{N}=4$ fields. $c_{\text {field }}= \pm 1$ determines the $\mathrm{SO}(4)$ representation for the massive fields, see (3.25) for $c_{\boldsymbol{B}},(3.36)$ for $c_{\boldsymbol{\psi}}$, and (B.2) for $c_{\boldsymbol{\lambda}}$.

The massless scalars in the effective theory (except for $\Sigma$ ) are most conveniently described by $\mathrm{SO}(5, n)$-rotated elements of the coset space

$$
\hat{\mathcal{V}}:=\langle\mathcal{V}\rangle^{-1} \mathcal{V}=\exp \left(\phi^{m a}\left[t_{m a}\right]\right)
$$

This is in contrast to the analysis of the massive scalar spectrum, for which it is efficient to consider the fluctuations $\phi^{m a}$ as it was done in the last section. Restricting to the $\mathcal{N}=2$ vector multiplets and truncating the massive modes $\phi^{\tilde{0} \hat{a}}$ and $\phi^{\hat{m} \tilde{a}}$, the only remaining 


\begin{tabular}{|l|l|l|l|}
\hline multiplets & fields & mass & charge \\
\hline 1 gravity & $g_{\mu \nu}, A_{\mu}^{\tilde{0}}, \psi_{\mu}^{\alpha}$ & 0 & 0 \\
\hline 1 gravitino & $\boldsymbol{\psi}_{\mu}, \boldsymbol{B}_{\mu \nu \alpha}$ & $\frac{1}{\sqrt{2}} \Sigma^{2} \gamma$ & $\gamma$ \\
\hline \multirow{2}{*}{$\left(1+n-2 n_{T}\right)$ vector } & $A_{\mu}^{0}, \chi_{\alpha}, \Sigma$ & 0 & 0 \\
\cline { 2 - 4 } & $A_{\mu}^{\tilde{a}}, \lambda_{\alpha}^{\tilde{a}}, \phi^{\tilde{0} \tilde{a}}$ & 0 & 0 \\
\hline \multirow{2}{*}{$n_{T}$ tensor } & $\boldsymbol{B}_{\mu \nu}^{\check{a}}, \lambda_{\alpha}^{\check{a}}, \phi^{\tilde{0} \check{a}}$ & $\frac{1}{\sqrt{2}} \Sigma^{2}\left|\gamma_{\check{a}}\right|$ & $\gamma_{\check{a}}$ \\
\hline \multirow{2}{*}{$n$ hyper } & $\lambda^{\tilde{a}}, \phi^{\alpha \tilde{a}}$ & $\frac{1}{\sqrt{2}} \Sigma^{2} \gamma$ & $\gamma$ \\
\cline { 2 - 4 } & $\lambda_{1,2}^{\check{a}}, \phi_{1,2}^{\alpha \check{a}}$ & $\frac{1}{\sqrt{2}} \Sigma^{2}\left|\gamma \mp \gamma_{\check{a}}\right|$ & $\gamma \mp \gamma_{\check{a}}$ \\
\hline
\end{tabular}

Table 3. $\mathcal{N}=2$ multiplets in the vacuum.

elements of the coset space are

$$
\hat{\mathcal{V}}_{\tilde{0}}^{\tilde{0}}, \quad \hat{\mathcal{V}}_{\tilde{0}}^{\tilde{a}}, \quad \hat{\mathcal{V}}_{\tilde{a}}^{\tilde{0}}, \quad \hat{\mathcal{V}}_{\tilde{a}}^{\tilde{b}}
$$

In fact, it turns out that all couplings involving the elements (4.17) can be expressed as functions of $\hat{\mathcal{V}}_{\tilde{0}}^{\tilde{a}}$ alone. In order to do that, one uses the relations

$$
\hat{\mathcal{V}}_{\tilde{0}}^{\tilde{o}}=\sqrt{1+\hat{\mathcal{V}}_{\tilde{0}}^{\tilde{a}} \hat{\mathcal{V}}_{\tilde{0} \tilde{a}}}, \quad \hat{\mathcal{V}}_{\tilde{a}}^{\tilde{0}}=\hat{\mathcal{V}}_{\tilde{0}}^{\tilde{a}}, \quad \hat{\mathcal{V}}_{\tilde{a}}^{\tilde{c}} \hat{\mathcal{V}}_{\tilde{b} \tilde{c}}=\delta_{\tilde{a} \tilde{b}}+\hat{\mathcal{V}}_{\tilde{a}}^{\tilde{0}} \hat{\mathcal{V}}_{\tilde{b}}^{\tilde{o}}, \quad \hat{\mathcal{V}}_{\tilde{a}}^{\tilde{b}} \hat{\mathcal{V}}_{\tilde{0} \tilde{b}}=\hat{\mathcal{V}}_{\tilde{0}}^{\tilde{0}} \hat{\mathcal{V}}_{\tilde{a}}^{\tilde{o}}
$$

The element $\hat{\mathcal{V}}_{\tilde{0}}^{\tilde{a}}$ itself can be expanded as

$$
\hat{\mathcal{V}}_{\tilde{0}}^{\tilde{a}}=\exp \left(\phi^{\tilde{0} \tilde{a}}\left[t_{\tilde{0} \tilde{a}}\right]\right)_{\tilde{0}}^{\tilde{a}},
$$

after truncating all massive modes. This implies, in particular, that $\hat{\mathcal{V}}_{\tilde{0}}^{\tilde{a}}$ has no dependence on $\phi^{\hat{m} \hat{a}}$. Therefore, the effective action of the scalars in the $\mathcal{N}=2$ vector multiplets decouples from the potentially massless scalars in the hypermultiplets as expected from $\mathcal{N}=2$ supersymmetry.

The reduced action then takes the simple form

$$
\begin{aligned}
& e^{-1} \mathcal{L}_{\text {class }}=-\frac{1}{2} R-H_{\Lambda \Sigma}^{a b} \partial_{\mu} h_{a}^{\Lambda} \partial^{\mu} h_{b}^{\Sigma}-\frac{3}{2} \Sigma^{-2} \partial_{\mu} \Sigma \partial^{\mu} \Sigma \\
& -\frac{1}{2}\left(\delta_{\tilde{a} \tilde{b}}-\frac{1}{1+\hat{\mathcal{V}}_{\tilde{0}}^{\tilde{c}} \hat{\mathcal{V}}_{\tilde{0} \tilde{c}}} \hat{\mathcal{V}}_{\tilde{0} \tilde{a}} \hat{\mathcal{V}}_{\tilde{0} \tilde{b}}\right) \partial_{\mu} \hat{\mathcal{V}}_{\tilde{0}}^{\tilde{a}} \partial^{\mu} \hat{\mathcal{V}}_{\tilde{0}}^{\tilde{b}} \\
& -\frac{1}{4} \Sigma^{-4} F_{\mu \nu}^{0} F^{\mu \nu 0}+\Sigma^{2} \sqrt{1+\hat{\mathcal{V}}_{\tilde{0}}^{\tilde{b}} \hat{\mathcal{V}}_{\tilde{0} \tilde{b}}} \hat{\mathcal{V}}_{\tilde{0} \tilde{a}} F_{\mu \nu}^{\tilde{0}} F^{\mu \nu \tilde{a}} \\
& -\frac{1}{4} \Sigma^{2}\left(3+2 \hat{\mathcal{V}}_{\tilde{0}}^{\tilde{a}} \hat{\mathcal{V}}_{\tilde{0} \tilde{a}}\right) F_{\mu \nu}^{\tilde{0}} F^{\mu \nu \tilde{0}}-\frac{1}{4} \Sigma^{2}\left(\delta_{\tilde{a} \tilde{b}}+2 \hat{\mathcal{V}}_{\tilde{0} \tilde{a}} \hat{\mathcal{V}}_{\tilde{0} \tilde{b}}\right) F_{\mu \nu}^{\tilde{a}} F^{\mu \nu \tilde{b}} \\
& +\frac{1}{4 \sqrt{2}} \epsilon^{\mu \nu \rho \sigma \tau} A_{\mu}^{0} F_{\nu \rho}^{\tilde{0}} F_{\sigma \tau}^{\tilde{0}}-\frac{1}{4 \sqrt{2}} \epsilon^{\mu \nu \rho \sigma \tau} A_{\mu}^{0} F_{\nu \rho}^{\tilde{a}} F_{\sigma \tau}^{\tilde{a}},
\end{aligned}
$$


where $H_{\Lambda \Sigma}^{a b}$ is the metric of the quaternionic manifold parametrized by the scalars in the massless hypermultiplets that we, however, do not discuss any further in this paper. Therefore, by comparison of (4.20) with (4.15) we find the identifications

$$
M^{0}=\frac{1}{\sqrt{2}} \Sigma^{2}, \quad M^{\tilde{0}}=\Sigma^{-1} \hat{\mathcal{V}}_{\tilde{0}}^{\tilde{0}}, \quad M^{\tilde{a}}=\Sigma^{-1} \hat{\mathcal{V}}_{\tilde{0}}^{\tilde{a}}
$$

and the real potential

$$
\mathcal{N}=\frac{1}{2} k_{0 \tilde{0} \tilde{0}} M^{0} M^{\tilde{0}} M^{\tilde{0}}+\frac{1}{2} k_{0 \tilde{a} \tilde{a}} M^{0} M^{\tilde{a}} M^{\tilde{a}}=\sqrt{2} M^{0} M^{\tilde{0}} M^{\tilde{0}}-\sqrt{2} M^{0} M^{\tilde{a}} M^{\tilde{a}} .
$$

This result specifies the constant tensors $k_{I J K}$ at the classical level. It is interesting to realize that the constraint $\mathcal{N} \stackrel{!}{=} 1$ translates with the identifications (4.21) into the condition (2.11) for the elements of the coset space. We conclude that the very special real manifold is the coset space

$$
\mathrm{SO}(1,1) \times \frac{\mathrm{SO}\left(1, n-2 n_{T}\right)}{\mathrm{SO}\left(n-2 n_{T}\right)}
$$

which is the subspace of (2.7) spanned by the massless scalars in the vector multiplets.

\subsection{One-loop effects and Chern-Simons terms}

In this section we determine the one-loop corrections to the gravity-vector sector of the $\mathcal{N}=2$ theory specified in subsection 4.2 . We focus on this sector, since the corrections due to integrating out massive fields are independent of the supersymmetry breaking scale and masses of the fields running in the loop. Let us stress that due to the preserved $\mathcal{N}=2$ supersymmetry and the fact that the Chern-Simons terms can only receive constant corrections, the integrating out process can only perturbatively correct the gravity-vector sector at the one-loop level.

To obtain the one-loop corrected $\mathcal{N}$ an analysis of the Chern-Simons terms is sufficient. The explicit loop computations were performed in [19, 20] and we can simply apply these results to our set-up. The studied Chern-Simons terms are of the form

$$
e^{-1} \mathcal{L}_{\mathrm{CS}}=\frac{1}{48} \epsilon^{\mu \nu \rho \sigma \tau} k_{I J K} A_{\mu}^{I} F_{\nu \rho}^{J} F_{\sigma \tau}^{K}+\frac{1}{16} \epsilon^{\mu \nu \rho \sigma \tau} k_{I} A_{\mu}^{I} R_{b \nu \rho}^{a} R_{a \sigma \tau}^{b} .
$$

Note that in [20] also the one-loop corrections to the gauge-gravitational Chern-Simons term, the second term in (4.24), were computed and we include the result for completeness. The one-loop corrections arise from integrating out massive fields that are charged under some gauge fields $A^{I}$. We denote the charges of massive gravitini, tensors, and spin- $1 / 2$ fermions collectively by $q_{I}$ and denote by $c_{\boldsymbol{\psi}}, c_{\boldsymbol{B}}$ and $c_{\boldsymbol{\lambda}}$ the \pm 1 choice of representation under the massive little group $\mathrm{SO}(4)$. The one-loop terms are calculated according to the table 4. These results hold when integrating out massive tensors, gravitini and spin- $1 / 2$ fermions with actions (3.24), (3.35), (B.1) and are thus readily applied to our supersymmetry breaking set-up.

Since all massive fields are only charged under $A^{0}$, the classical terms in (4.22) are unmodified. The fully quantum corrected result therefore reads

$$
k_{0 \tilde{0} \tilde{0}}=-k_{0 \tilde{a} \tilde{a}}=2 \sqrt{2} .
$$




\begin{tabular}{|cccc|}
\hline & spin $1 / 2$ & tensor & spin $3 / 2$ \\
\hline$k_{I J K}=$ & $\frac{1}{2} q_{I} q_{J} q_{K} \cdot c_{\boldsymbol{\lambda}}$ & $-2 q_{I} q_{J} q_{K} \cdot c_{\boldsymbol{B}}$ & $\frac{5}{2} q_{I} q_{J} q_{K} \cdot c_{\boldsymbol{\psi}}$ \\
$k_{I}=$ & $-q_{I} \cdot c_{\boldsymbol{\lambda}}$ & $-8 q_{I} \cdot c_{\boldsymbol{B}}$ & $19 q_{I} \cdot c_{\boldsymbol{\psi}}$ \\
\hline
\end{tabular}

Table 4. One-loop corrections due to integrating out massive spin- $1 / 2$ fermions, tensors, and spin-3/2 fermions.

Using table 4 we find that the massive states summarized in table 2 induce by the one-loop couplings

$$
k_{000}=\frac{1}{2}\left[\left(13-n+2 n_{T}\right) \gamma^{3}-2 \sum_{\breve{a}}\left|\gamma_{\breve{a}}\right|^{3}+\sum_{\breve{a}}\left|\gamma-\gamma_{\breve{a}}\right|^{3}+\sum_{\breve{a}}\left|\gamma+\gamma_{\breve{a}}\right|^{3}\right] .
$$

Furthermore, we find the gravitational one-loop Chern-Simons terms

$$
k_{0}=-\left[\left(-35-n+2 n_{T}\right) \gamma+10 \sum_{\breve{a}}\left|\gamma_{\breve{a}}\right|+\sum_{\breve{a}}\left|\gamma-\gamma_{\breve{a}}\right|+\sum_{\check{a}}\left|\gamma+\gamma_{\check{a}}\right|\right] \text {. }
$$

The existence of new Chern-Simons couplings implies that the effective theory still sees remnants of the underlying $\mathcal{N}=4$ theory at arbitrarily low energy scales. In fact, the mass of the fields listed in table 3 can be made arbitrarily large by choosing the VEV of the modulus $\Sigma$. The constants $\gamma$ and $\gamma_{a}$ appearing in (4.26) and (4.27) are the imaginary parts of the eigenvalues of $\xi^{M N}$ and therefore independent of the VEVs of the fields.

\section{Conclusions}

In this work we have studied partial supersymmetry breaking in $\mathcal{N}=4$ supergravity theories. We focused on a certain subclass of gauged supergravity theories that possess tensor fields with first order kinetic terms. These are characterized by a non-trivial embedding tensor $\xi_{M N}$. The presence of these couplings also ensures that they can become massive by a tensorial Higgs mechanism and are gauged by a distinctive U(1) vector field $A^{0}$. The Higgs mechanism allows that the tensors acquire three degrees of freedom by absorbing a dynamical vector. Clearly, this is just a special case of the mechanisms that are employed to construct general supergravity theories using tensor hierarchies $[12,35]$. We show that the presence of these couplings permits the existence of simple $\mathcal{N}=2$ supersymmetric Minkowski vacua.

The fluctuations around the $\mathcal{N}=2$ supersymmetric Minkowski vacua are split into massive and massless $\mathcal{N}=2$ multiplets. In fact, starting with the $\mathcal{N}=4$ gravity multiplet and $n$ vector multiplets, we showed that the massless $\mathcal{N}=2$ spectrum consists of the gravity multiplet, $n-2 n_{T}+1$ vector multiplets, and $n_{H}$ hypermultiplets. In addition one finds a massive spin-3/2 multiplet, $n_{T}$ complex massive tensor multiplets, and $n-n_{H}$ massive hypermultiplets. The massive spin- $3 / 2$ multiplet contains in addition to the gravitino degrees of freedom also two complex massive tensors. The number $n_{T}$ is given by $2 n_{T}=$ $\operatorname{rank}\left(\xi_{M N}\right)-4$, where the 4 corresponds to the massive tensors in the spin- $3 / 2$ multiplet. 
The degeneracy $n_{H}$ is given by the number of hypermultiplets that satisfy the masslessness condition (4.11).

We also determined the $\mathcal{N}=2$ low-energy effective action for the massless modes particularly focusing on the vector multiplets. In order to do that we first extracted the classical $\mathcal{N}=2$ couplings obtained by dropping all massive fields. We argued, however, that it is crucial to evaluate the actions for the massive tensors, and massive spin- $3 / 2$ and spin- $1 / 2$ fermions that are charged under the vector field $A^{0}$. This is due to the fact, that these massive fields induce corrections to the kinetic terms and Chern-Simons terms of $A^{0}$ at one-loop. Crucially, these corrections are independent of the supersymmetry breaking scale and thus have to be included despite the fact that they are of loop order. In other words, from the Chern-Simons terms of the effective theory, one can extract information about the underlying $\mathcal{N}=4$ theory. This is of similar spirit as the discussion in [36], where five-dimensional one-loop Chern-Simons terms were used to extract information about an underling six-dimensional theory.

Let us briefly discuss possible applications of our results and comment of further extensions. Five-dimensional theories with $\mathcal{N}=4$ supersymmetry can arise, for example, in compactifications of M-theory on $K 3 \times T^{2}$, or Type IIB supergravity on $K 3 \times S^{1}$. In order to obtain a gauged $\mathcal{N}=4$ theory the $T^{2}$ or $S^{1}$ reduction can, for example, be made non-trivial by demanding Scherk-Schwarz boundary conditions. In fact, in such reductions one expects that certain states are charged under the Kaluza-Klein vector that is identified with our $A^{0}$. In particular, it would be interesting to carry out a Scherk-Schwarz reduction of a six-dimensional $(2,0)$ theory on a circle and determine its $\mathcal{N}=2$ vacua.

More involved five-dimensional gauged $\mathcal{N}=4$ supergravity theories arise from Mtheory on a general SU(2) structure manifold, or from Type IIB on a squashed SasakiEinstein manifold [23-30]. These theories in general also admit non-Abelian gaugings and allow for non-trivial Anti-de Sitter vacua. In a future project we hope to extend our analysis to include these more involved situations. We expect that similar to our discussion for the Minkowski vacua also one-loop effects will be essential when evaluating the effective theory.

\section{Acknowledgments}

We would like to thank Federico Bonetti, Stefan Hohenegger, Jan Keitel, Jan Louis, Severin Lüst, Tom Pugh, and Hagen Triendl for interesting discussions and comments. This work was supported by a research grant of the Max Planck Society.

\section{A Conventions and identities}

\section{A.1 Spacetime conventions}

In this section we state the conventions of Riemannian geometry adopted in this paper. We denote curved five-dimensional spacetime indices by Greek letters $\mu, \nu, \ldots$ Antisymmetrizations of any kind of indices are made with weight one, i.e. include a factor of $1 / n$ ! . For the five-dimensional metric $g_{\mu \nu}$ we use the $(-,+,+,+,+)$ sign convention and we 
choose a negative sign in front of the Einstein-Hilbert term. Furthermore we set

$$
\kappa^{2}=1
$$

We adopt the following convention for the Levi-Civita tensor with curved indices $\epsilon_{\mu \nu \rho \lambda \sigma}$, $\epsilon^{\mu \nu \rho \lambda \sigma}$

$$
\epsilon_{01234}=+e, \quad, \epsilon^{01234}=-e^{-1},
$$

where $e=\sqrt{-\operatorname{det} g_{\mu \nu}}$.

We denote the five-dimensional spacetime gamma matrices by $\gamma_{\mu}$, satisfying

$$
\left\{\gamma_{\mu}, \gamma_{\nu}\right\}=2 g_{\mu \nu}
$$

Antisymmetrized products of gamma matrices are defined as

$$
\gamma_{\mu_{1}, \ldots, \mu_{k}}:=\gamma_{\left[\mu_{1}\right.} \gamma_{\mu_{2}} \ldots \gamma_{\left.\mu_{k}\right]}
$$

The charge conjugation matrix $C$ is chosen such that

$$
C^{T}=-C=C^{-1}
$$

and it fulfills the relation

$$
C \gamma_{\mu} C^{-1}=\left(\gamma_{\mu}\right)^{T}
$$

All massless spinors in this paper are symplectic Majorana. In the $\mathcal{N}=4$ theory they are subject to the condition

$$
\bar{\chi}^{i}:=\left(\chi_{i}\right)^{\dagger} \gamma_{0}=\Omega^{i j} \chi_{j}^{T} C,
$$

where $i, j=1, \ldots, 4$ and $\Omega^{i j}$ is the USp(4) symplectic form defined in (2.2). The $\mathcal{N}=2$ symplectic Majorana condition reads

$$
\bar{\chi}^{\alpha}:=\left(\chi_{\alpha}\right)^{\dagger} \gamma_{0}=\varepsilon^{\alpha \beta} \chi_{\beta}^{T} C,
$$

where $\alpha, \beta=1,2$ and $\varepsilon^{\alpha \beta}$ is the two-dimensional epsilon tensor. Let us state a set of useful relations between bilinears of symplectic Majorana spinors

$$
\bar{\chi}^{i} \gamma_{\mu_{1} \ldots \mu_{k}} \lambda^{j}= \begin{cases}+\bar{\lambda}^{j} \gamma_{\mu_{1} \ldots \mu_{k}} \chi^{i} & (k=0,1,4,5) \\ -\bar{\lambda}^{j} \gamma_{\mu_{1} \ldots \mu_{k}} \chi^{i} & (k=2,3)\end{cases}
$$

and

$$
\bar{\chi}^{i} \gamma_{\mu_{1} \ldots \mu_{k}} \lambda_{i}= \begin{cases}-\bar{\lambda}^{i} \gamma_{\mu_{1} \ldots \mu_{k}} \chi_{i} & (k=0,1,4,5) \\ +\bar{\lambda}^{i} \gamma_{\mu_{1} \ldots \mu_{k}} \chi_{i} & (k=2,3) .\end{cases}
$$

Both relations are are also true for symplectic Majorana spinors carrying indices $\alpha, \beta=1,2$. 


\section{A.2 $\mathrm{SO}(5)$ gamma matrices}

The scalars in the vector multiplets of the $\mathcal{N}=4$ theory span the coset manifold

$$
\mathcal{M}_{5, n}=\frac{\mathrm{SO}(5, n)}{\mathrm{SO}(5) \times \mathrm{SO}(n)} .
$$

The coset representatives are denoted by $\left(\mathcal{V}_{M}{ }^{m}, \mathcal{V}_{M}{ }^{a}\right)$ and their properties are discussed in subsection 2.1. In this section we collect some properties of the $\mathrm{SO}(5)$ gamma matrices $\Gamma_{m}^{i j}$, which satisfy

$$
\left\{\Gamma_{m}, \Gamma_{n}\right\}=2 \delta_{m n} \mathbf{1}_{4}
$$

Furthermore we have

$$
\Gamma_{m}^{i j}=-\Gamma_{m}^{j i}, \quad \Gamma_{m i}{ }^{i}=0, \quad\left(\Gamma_{m}^{i j}\right)^{*}=\Omega_{i k} \Omega_{j l} \Gamma_{m}^{k l}
$$

as well as

$$
\Gamma^{m i j} \Gamma_{m k l}=4 \delta_{k l}^{i j}-\Omega^{i j} \Omega_{k l} .
$$

Note that one can use $\Gamma_{m n}:=\Gamma_{[m} \Gamma_{n]}$ in order to switch between antisymmetric tensors $T_{m n}$ and symmetric tensors $T_{i j}$ in the following way

$$
T_{i j}=T_{m n} \Gamma_{i j}^{m n}, \quad T_{m n}=\frac{1}{8} \Gamma_{m n i j} T^{i j},
$$

which can be checked using the identity

$$
\Gamma_{m n i j} \Gamma^{p q i j}=8 \delta_{m n}^{p q}
$$

This correspondence is for example used in (2.25).

\section{B Derivation of the mass terms and couplings}

In this section we explicitly derive the masses for the spin- $1 / 2$ fermions and scalars induced by the supergravity breaking. We also evaluate the charges of the spin- $1 / 2$ fermions under the Abelian gauge field $A^{0}$.

We shortly note that the Lagrangian of a massive spin-1/2 Dirac spinor is given by

$$
e^{-1} \mathcal{L}_{\boldsymbol{\lambda}}=-\overline{\boldsymbol{\lambda}} \not \boldsymbol{D} \boldsymbol{\lambda}+c_{\boldsymbol{\lambda}} m \overline{\boldsymbol{\lambda}} \boldsymbol{\lambda}, \quad c_{\boldsymbol{\lambda}}= \pm 1 .
$$

The $c_{1 / 2}$ in (B.1) refers to the two inequivalent spinor representations of the massive little group, in detail

$$
\begin{aligned}
& c_{\boldsymbol{\lambda}}=+1 \Leftrightarrow\left(\frac{1}{2}, 0\right) \text { of } \mathrm{SO}(4) \cong \mathrm{SU}(2) \times \mathrm{SU}(2) \\
& c_{\boldsymbol{\lambda}}=-1 \Leftrightarrow\left(0, \frac{1}{2}\right) \text { of } \mathrm{SO}(4) \cong \mathrm{SU}(2) \times \mathrm{SU}(2) .
\end{aligned}
$$

The mass of the physical mode is given by $m$. 


\section{B.1 Fermion masses}

Let us now investigate the masses the $\mathcal{N}=4$ gaugini acquire from supersymmetry breaking. The mass terms read

$$
\begin{aligned}
e^{-1} \mathcal{L}_{\lambda, \text { mass }}= & i\left(\frac{1}{2 \sqrt{2}} \Sigma^{2} \xi_{a b} \delta_{i}^{j}-\frac{3}{2 \sqrt{6}} A_{1 i}{ }^{j} \delta_{a b}\right) \bar{\lambda}^{i a} \lambda_{j}^{b}= \\
= & 0 \cdot \bar{\lambda}^{\alpha \tilde{a}} \lambda_{\alpha}^{\tilde{b}}+\frac{1}{2 \sqrt{2}} i \Sigma^{2} \xi_{\hat{a} \hat{b}} \bar{\lambda}^{\alpha \hat{a}} \lambda_{\alpha}^{\hat{b}}-\frac{3}{2 \sqrt{6}} i A_{1 \dot{\alpha}} \dot{\beta}_{\tilde{a} \tilde{b}} \bar{\lambda}^{\dot{\alpha} \tilde{a}} \lambda_{\dot{\beta}}^{\tilde{b}} \\
& i\left(\frac{1}{2 \sqrt{2}} \Sigma^{2} \xi_{\hat{a} \hat{b}} \delta_{\dot{\alpha}}^{\dot{\beta}}-\frac{3}{2 \sqrt{6}} A_{1 \dot{\alpha}}{ }^{\dot{\beta}} \delta_{\hat{a} \hat{b}}\right) \bar{\lambda}^{\dot{\alpha} \hat{a}} \lambda_{\dot{\beta}}^{\hat{b}} .
\end{aligned}
$$

Let us discuss the four different types of fields separately.

1. $\lambda_{\alpha}^{\tilde{a}}$. We observe that the $\lambda_{\alpha}^{\tilde{a}}$ stay massless. Thus we have $2\left(n-2 n_{T}\right)$ massless spin- $1 / 2$ fermions supplemented by a symplectic Majorana condition.

2. $\lambda_{\alpha}^{\hat{a}}$. For the fermions $\lambda_{\alpha}^{\hat{a}}$. We write the mass terms using the split (4.7) as

$$
\begin{aligned}
\frac{1}{2 \sqrt{2}} i \Sigma^{2} \xi_{\hat{a} \hat{b}} \bar{\lambda}^{\alpha \hat{a}} \lambda_{\alpha}^{\hat{b}} & =\frac{1}{2 \sqrt{2}} i \Sigma^{2} \sum_{\check{a}} \gamma_{\check{a}} \varepsilon_{k l} \bar{\lambda}^{\alpha[\check{a} k]} \lambda_{\alpha}^{[\check{a} l]} \\
& =\frac{1}{\sqrt{2}} \Sigma^{2} \sum_{\check{a}} \gamma_{\breve{a}} \overline{\boldsymbol{\lambda}}^{\alpha \check{a}} \lambda_{\alpha}^{\check{a}},
\end{aligned}
$$

with $m, n$ both taking values 1,2 . Here we redefined the fermions by introducing $\boldsymbol{\lambda}_{\alpha}^{\check{a}}$ as in (4.8a) and drop the symplectic Majorana condition, such that the mass terms become diagonal. Let us now have a look how the corresponding kinetic terms transform under this redefinition

$$
-\frac{1}{2} \sum_{\breve{a}}\left(\bar{\lambda}^{\alpha[\check{a} 1]} \not \partial \lambda_{\alpha}^{[\check{a} 1]}+\bar{\lambda}^{\alpha[\check{a} 2]} \not \partial \lambda_{\alpha}^{[\check{a} 2]}\right)=-\sum_{\breve{a}} \bar{\lambda}^{\alpha \check{a}} \not \partial \lambda_{\alpha}^{\check{a}}
$$

The computation of the charge under $A^{0}$ proceeds as for the mass terms. By comparing the action with (B.1) we find

$$
m_{\boldsymbol{\lambda}_{\alpha}^{\check{a}}}=\frac{1}{\sqrt{2}} \Sigma^{2}\left|\gamma_{\check{a}}\right|, \quad c_{\boldsymbol{\lambda}_{\alpha}^{\check{a}}}=\operatorname{sign} \gamma_{\alpha}, \quad q_{\boldsymbol{\lambda}_{\alpha}^{\check{a}}}=\gamma_{\check{a}} .
$$

3. $\lambda_{\dot{\alpha}}^{\tilde{a}}$. The structure of mass terms of the fermions $\lambda_{\dot{\alpha}}^{\tilde{a}}$ is similar to those of the gravitino masses. In particular, the diagonalization procedure of the gravitino mass terms automatically diagonalizes the mass terms of the $\lambda_{\dot{\alpha}}^{\tilde{a}}$. Again we move from symplectic Majorana spinors to Dirac spinors $\boldsymbol{\lambda}^{\tilde{a}}$ using (4.8b). We find

$$
m_{\boldsymbol{\lambda}^{\tilde{a}}}=\frac{1}{\sqrt{2}} \Sigma^{2} \gamma, \quad c_{\boldsymbol{\lambda}^{\tilde{a}}}=-1, \quad q_{\boldsymbol{\lambda}^{\tilde{a}}}=\gamma .
$$

The $\boldsymbol{\lambda}^{\tilde{a}}$ are in the $\left(0, \frac{1}{2}\right)$ representation of $\mathrm{SO}(4)$. 
4. $\lambda_{\dot{\alpha}}^{\hat{a}}$. The mass terms become after the split (4.7)

$$
i \sum_{\check{a}}\left(\frac{1}{2 \sqrt{2}} \Sigma^{2} \gamma_{\check{a}} \varepsilon_{k l} \delta_{\dot{\alpha}}^{\dot{\beta}}-\frac{3}{2 \sqrt{6}} A_{1 \dot{\alpha}} \dot{\beta} \delta_{k l}\right) \bar{\lambda}^{\dot{\alpha}[\check{a} k]} \lambda_{\dot{\beta}}^{[\check{a} l]} .
$$

We redefine and use Dirac spinors $\boldsymbol{\lambda}_{1}^{\check{a}}$ and $\boldsymbol{\lambda}_{2}^{\check{a}}$ given in $(4.8 \mathrm{~b})$. The mass terms then become

$$
\sum_{\breve{a}}\left[\frac{1}{\sqrt{2}} \Sigma^{2} \gamma_{\check{a}}\left(\overline{\boldsymbol{\lambda}}_{1}^{\check{a}} \boldsymbol{\lambda}_{1}^{\check{a}}-\overline{\boldsymbol{\lambda}}_{2}^{\check{a}} \boldsymbol{\lambda}_{2}^{\check{a}}\right)-\frac{1}{\sqrt{2}} \Sigma^{2} \gamma\left(\overline{\boldsymbol{\lambda}}_{1}^{\check{a}} \boldsymbol{\lambda}_{1}^{\check{a}}+\overline{\boldsymbol{\lambda}}_{2}^{\check{a}} \boldsymbol{\lambda}_{2}^{\check{a}}\right)\right] .
$$

The kinetic terms are unaffected. We conclude that

$$
\begin{aligned}
& m_{\boldsymbol{\lambda}_{1}^{\check{a}}}=\frac{1}{\sqrt{2}} \Sigma^{2}\left|\gamma_{\check{a}}-\gamma\right|, \quad m_{\boldsymbol{\lambda}_{2}^{\check{a}}}=\frac{1}{\sqrt{2}} \Sigma^{2}\left|-\gamma_{\check{a}}-\gamma\right|, \\
& c_{\boldsymbol{\lambda}_{1}^{\check{a}}}=\operatorname{sign}\left(\gamma_{\breve{a}}-\gamma\right), \quad c_{\boldsymbol{\lambda}_{2}^{\check{a}}}=\operatorname{sign}\left(-\gamma_{\check{a}}-\gamma\right), \\
& q_{\boldsymbol{\lambda}_{1}^{\check{a}}}=\gamma_{\check{a}}-\gamma, \quad \quad q_{\boldsymbol{\lambda}_{2}^{\check{a}}}=-\gamma_{\check{a}}-\gamma .
\end{aligned}
$$

\section{B.2 Scalar masses}

Lastly we investigate the scalar degrees of freedom in the vacuum (except of $\Sigma$ ). In order to derive the scalar masses we insert the expansion (4.4) into the scalar potential (2.26)

$$
\begin{aligned}
e^{-1} \mathcal{L}_{\text {pot }}= & -\frac{1}{16} \xi^{M N} \xi^{P Q} \Sigma^{4}\left[\langle\mathcal{V}\rangle \exp \left(\sum_{m, a} \phi^{m a}\left[t_{m a}\right]\right) \exp \left(\sum_{n, b} \phi^{n b}\left[t_{n b}\right]\right)^{T}\langle\mathcal{V}\rangle^{T}\right]_{M P} \times \\
& {\left[\langle\mathcal{V}\rangle \exp \left(\sum_{p, c} \phi^{p c}\left[t_{p c}\right]\right) \exp \left(\sum_{q, d} \phi^{q d}\left[t_{q d}\right]\right)^{T}\langle\mathcal{V}\rangle^{T}\right]_{N Q} \cdot }
\end{aligned}
$$

To read off the mass terms of the scalars, we focus on the terms quadratic in $\phi^{m a}$

$$
e^{-1} \mathcal{L}_{\phi, \text { mass }}=-\frac{1}{16} \Sigma^{4} \phi^{m a} \phi^{n b}\left(8 \xi_{m n} \xi_{a b}+4 \delta_{m n} \xi_{a c} \xi_{b}{ }^{c}+4 \delta_{a b} \xi_{m p} \xi_{n}{ }^{p}\right) .
$$

According to the index split (4.3) the scalar fields arrange in four different groups:

1. $\phi^{\tilde{0} \tilde{a}}$. The mass terms for these fields vanish:

$$
e^{-1} \mathcal{L}_{\phi, \text { mass }}=0 \text {. }
$$

Thus we find $n-2 n_{T}$ massless real scalar fields $\phi^{\tilde{0} \tilde{a}}$.

2. $\phi^{\hat{m} \tilde{a}}$. The mass terms of these modes receive one contribution from the gauging $\xi^{m n}$

$$
e^{-1} \mathcal{L}_{\phi, \text { mass }}=-\frac{1}{4} \gamma^{2} \Sigma^{4} \phi^{\hat{m} \tilde{a}} \phi_{\hat{m} \tilde{a}} .
$$

We can now complexify the scalars as in (4.6a) into the $2\left(n-2 n_{T}\right)$ massive complex scalars $\phi^{\alpha \tilde{a}}$ with mass ${ }^{8}$

$$
m_{\phi^{\alpha \tilde{a}}}=\frac{1}{\sqrt{2}} \Sigma^{2} \gamma .
$$

${ }^{8}$ We stress that the kinetic terms for the scalars here and in the following are always automatically canonically normalized, even after the field redefinitions carried out in this section. This one can check explicitly by inserting the expansion (4.4) into the $\mathcal{N}=4$ scalar kinetic terms. 
3. $\phi^{\tilde{0} \hat{a}}$. There is now solely a mass contribution from the gaugings $\xi^{a b}$

$$
e^{-1} \mathcal{L}_{\phi, \text { mass }}=-\frac{1}{4} \Sigma^{4} \sum_{\hat{a}} \gamma_{\tilde{a}}^{2} \phi^{\tilde{0} \hat{a}} \phi^{\tilde{0} \hat{a}}
$$

Using the definition (4.6a) one identifies $n_{T}$ massive complex scalar fields $\phi^{\tilde{0} a}$ with mass

$$
m_{\phi^{\tilde{0} \check{a}}}=\frac{1}{\sqrt{2}} \Sigma^{2}\left|\gamma_{\tilde{a}}\right|
$$

4. $\phi^{\hat{m} \hat{a}}$. We now face mass contributions both from $\xi^{m n}$ and $\xi^{a b}$

$$
e^{-1} \mathcal{L}_{\phi, \text { mass }}=-\frac{1}{16} \Sigma^{4} \sum_{\breve{a}, \alpha}\left(8 \gamma \gamma_{\check{a}} \varepsilon_{\dot{\alpha} \dot{\beta}} \varepsilon_{k l}+4 \gamma^{2} \delta_{\dot{\alpha} \dot{\beta}} \delta_{k l}+4 \gamma_{\breve{a}}^{2} \delta_{\dot{\alpha} \dot{\beta}} \delta_{k l}\right) \phi^{[\alpha \dot{\alpha}][\check{a} k]} \phi^{[\alpha \dot{\beta}][\check{a} l]} .
$$

One can check that the mass terms are diagonalized by the redefinitions (4.6b) and (4.6c) to $4 n_{T}$ complex scalars $\phi_{1}^{\alpha \check{a}}$ and $\phi_{2}^{\alpha \breve{a}}$ with masses

$$
m_{\phi_{1}^{\alpha \check{a}}}=\frac{1}{\sqrt{2}} \Sigma^{2}\left|\gamma-\gamma_{\check{a}}\right|, \quad m_{\phi_{2}^{\alpha \check{a}}}=\frac{1}{\sqrt{2}} \Sigma^{2}\left|\gamma+\gamma_{\check{a}}\right| .
$$

Open Access. This article is distributed under the terms of the Creative Commons Attribution License (CC-BY 4.0), which permits any use, distribution and reproduction in any medium, provided the original author(s) and source are credited.

\section{References}

[1] S. Cecotti, L. Girardello and M. Porrati, Two into one won't go, Phys. Lett. B 145 (1984) 61 [INSPIRE].

[2] S. Cecotti, L. Girardello and M. Porrati, Constraints on partial superhiggs, Nucl. Phys. B 268 (1986) 295 [inSPIRE].

[3] S. Cecotti, L. Girardello and M. Porrati, An Exceptional $N=2$ Supergravity With Flat Potential and Partial Superhiggs, Phys. Lett. B 168 (1986) 83 [InSPIRE].

[4] S. Ferrara, L. Girardello and M. Porrati, Minimal Higgs branch for the breaking of half of the supersymmetries in $N=2$ supergravity, Phys. Lett. B 366 (1996) 155 [hep-th/9510074] [INSPIRE].

[5] I. Antoniadis, H. Partouche and T.R. Taylor, Spontaneous breaking of $N=2$ global supersymmetry, Phys. Lett. B 372 (1996) 83 [hep-th/9512006] [INSPIRE].

[6] P. Fré, L. Girardello, I. Pesando and M. Trigiante, Spontaneous $N=2 \rightarrow N=1$ local supersymmetry breaking with surviving compact gauge group, Nucl. Phys. B 493 (1997) 231 [hep-th/9607032] [INSPIRE].

[7] E. Kiritsis and C. Kounnas, Perturbative and nonperturbative partial supersymmetry breaking: $N=4 \rightarrow N=2 \rightarrow N=1$, Nucl. Phys. B 503 (1997) 117 [hep-th/9703059] [INSPIRE].

[8] L. Andrianopoli, R. D'Auria, S. Ferrara and M.A. Lledó, Super Higgs effect in extended supergravity, Nucl. Phys. B 640 (2002) 46 [hep-th/0202116] [INSPIRE]. 
[9] J. Louis, P. Smyth and H. Triendl, Spontaneous $N=2 \rightarrow N=1$ Supersymmetry Breaking in Supergravity and Type II String Theory, JHEP 02 (2010) 103 [arXiv:0911.5077] [InSPIRE].

[10] J. Louis, P. Smyth and H. Triendl, The $N=1$ Low-Energy Effective Action of Spontaneously Broken $N=2$ Supergravities, JHEP 10 (2010) 017 [arXiv:1008.1214] [INSPIRE].

[11] T. Hansen and J. Louis, Examples of $\mathcal{N}=2 \rightarrow \mathcal{N}=1$ supersymmetry breaking, JHEP 11 (2013) 075 [arXiv: 1306.5994] [INSPIRE].

[12] H. Samtleben, Lectures on Gauged Supergravity and Flux Compactifications, Class. Quant. Grav. 25 (2008) 214002 [arXiv:0808.4076] [INSPIRE].

[13] B. de Wit, H. Samtleben and M. Trigiante, On Lagrangians and gaugings of maximal supergravities, Nucl. Phys. B 655 (2003) 93 [hep-th/0212239] [INSPIRE].

[14] B. de Wit, H. Samtleben and M. Trigiante, Magnetic charges in local field theory, JHEP 09 (2005) 016 [hep-th/0507289] [INSPIRE].

[15] O. Hohm and J. Louis, Spontaneous $N=2 \rightarrow N=1$ supergravity breaking in three dimensions, Class. Quant. Grav. 21 (2004) 4607 [hep-th/0403128] [INSPIRE].

[16] P.K. Townsend, K. Pilch and P. van Nieuwenhuizen, Selfduality in Odd Dimensions, Phys. Lett. B 136 (1984) 38 [Addendum ibid. B 137 (1984) 443] [INSPIRE].

[17] G. Dall'Agata, C. Herrmann and M. Zagermann, General matter coupled $N=4$ gauged supergravity in five-dimensions, Nucl. Phys. B 612 (2001) 123 [hep-th/0103106] [INSPIRE].

[18] J. Schon and M. Weidner, Gauged N=4 supergravities, JHEP 05 (2006) 034 [hep-th/0602024] [INSPIRE].

[19] F. Bonetti, T.W. Grimm and S. Hohenegger, A Kaluza-Klein inspired action for chiral p-forms and their anomalies, Phys. Lett. B 720 (2013) 424 [arXiv:1206.1600] [INSPIRE].

[20] F. Bonetti, T.W. Grimm and S. Hohenegger, One-loop Chern-Simons terms in five dimensions, JHEP 07 (2013) 043 [arXiv: 1302.2918] [INSPIRE].

[21] E. Witten, Phase transitions in M-theory and F-theory, Nucl. Phys. B 471 (1996) 195 [hep-th/9603150] [INSPIRE].

[22] D.R. Morrison and N. Seiberg, Extremal transitions and five-dimensional supersymmetric field theories, Nucl. Phys. B 483 (1997) 229 [hep-th/9609070] [InSPIRE].

[23] J.P. Gauntlett, D. Martelli, J. Sparks and D. Waldram, Supersymmetric AdS $S_{5}$ solutions of M-theory, Class. Quant. Grav. 21 (2004) 4335 [hep-th/0402153] [INSPIRE].

[24] D. Cassani, G. Dall'Agata and A.F. Faedo, Type IIB supergravity on squashed Sasaki-Einstein manifolds, JHEP 05 (2010) 094 [arXiv: 1003.4283] [INSPIRE].

[25] J.P. Gauntlett and O. Varela, Universal Kaluza-Klein reductions of type IIB to $N=4$ supergravity in five dimensions, JHEP 06 (2010) 081 [arXiv:1003.5642] [INSPIRE].

[26] J.T. Liu, P. Szepietowski and Z. Zhao, Consistent massive truncations of IIB supergravity on Sasaki-Einstein manifolds, Phys. Rev. D 81 (2010) 124028 [arXiv:1003.5374] [INSPIRE].

[27] D. Cassani and A.F. Faedo, A supersymmetric consistent truncation for conifold solutions, Nucl. Phys. B 843 (2011) 455 [arXiv: 1008.0883] [INSPIRE].

[28] I. Bena, G. Giecold, M. Graña, N. Halmagyi and F. Orsi, Supersymmetric Consistent Truncations of IIB on $T^{1,1}$, JHEP 04 (2011) 021 [arXiv: 1008.0983] [INSPIRE]. 
[29] I. Bah, A. Faraggi, J.I. Jottar and R.G. Leigh, Fermions and Type IIB Supergravity On Squashed Sasaki-Einstein Manifolds, JHEP 01 (2011) 100 [arXiv:1009.1615] [INSPIRE].

[30] E. O Colgain and O. Varela, Consistent reductions from D = 11 beyond Sasaki-Einstein, Phys. Lett. B 703 (2011) 180 [arXiv:1106.4781] [InSPIRE].

[31] D. Cassani, G. Dall'Agata and A.F. Faedo, BPS domain walls in $N=4$ supergravity and dual flows, JHEP 03 (2013) 007 [arXiv: 1210.8125] [INSPIRE].

[32] R.A. Horn and C.R. Johnson, Matrix Analysis, Cambridge University Press, Cambridge, U.K. (1990).

[33] C.M. Hull, BPS supermultiplets in five-dimensions, JHEP 06 (2000) 019 [hep-th/0004086] [INSPIRE].

[34] C. Horst, J. Louis and P. Smyth, Electrically gauged $N=4$ supergravities in $D=4$ with $N=2$ vacua, JHEP 03 (2013) 144 [arXiv:1212.4707] [INSPIRE].

[35] J. Hartong and T. Ortín, Tensor Hierarchies of 5- and 6-Dimensional Field Theories, JHEP 09 (2009) 039 [arXiv:0906.4043] [INSPIRE].

[36] F. Bonetti, T.W. Grimm and S. Hohenegger, Exploring $6 D$ origins of $5 D$ supergravities with Chern-Simons terms, JHEP 05 (2013) 124 [arXiv: 1303.2661] [INSPIRE]. 